\title{
MassGenie: A Transformer-Based Deep Learning Method for Identifying Small Molecules from Their Mass Spectra
}

Shrivastava, Aditya Divyakant; Swainston, Neil; Samanta, Soumitra; Roberts, Ivayla; Wright Muelas, Marina; Kell, Douglas

\section{Published in:}

Biomolecules

Link to article, DOI:

10.3390/biom11121793

Publication date:

2021

Document Version

Publisher's PDF, also known as Version of record

Link back to DTU Orbit

Citation (APA):

Shrivastava, A. D., Swainston, N., Samanta, S., Roberts, I., Wright Muelas, M., \& Kell, D. (2021). MassGenie: A Transformer-Based Deep Learning Method for Identifying Small Molecules from Their Mass Spectra.

Biomolecules, 11(12), [1793]. https://doi.org/10.3390/biom11121793

\section{General rights}

Copyright and moral rights for the publications made accessible in the public portal are retained by the authors and/or other copyright owners and it is a condition of accessing publications that users recognise and abide by the legal requirements associated with these rights.

- Users may download and print one copy of any publication from the public portal for the purpose of private study or research.

- You may not further distribute the material or use it for any profit-making activity or commercial gain

- You may freely distribute the URL identifying the publication in the public portal 


\title{
MassGenie: A Transformer-Based Deep Learning Method for Identifying Small Molecules from Their Mass Spectra
}

\author{
Aditya Divyakant Shrivastava ${ }^{1,2}$, Neil Swainston ${ }^{1,3}$, Soumitra Samanta ${ }^{1}$, Ivayla Roberts ${ }^{1}$, \\ Marina Wright Muelas ${ }^{1}$ and Douglas B. Kell ${ }^{1,3,4, *(\mathbb{D})}$
}

1 Department of Biochemistry and Systems Biology, Institute of Systems, Molecular and Integrative Biology, Faculty of Health and Life Sciences, University of Liverpool, Crown St, Liverpool L69 7ZB, UK; aditya@mellizyme.com (A.D.S.); neil@mellizyme.com (N.S.); ssamanta@liv.ac.uk (S.S.); ivayla.roberts@liv.ac.uk (I.R.); m.wright-muelas@liv.ac.uk (M.W.M.)

2 Department of Computer Science and Engineering, Nirma University, Ahmedabad 382481, India

3 Mellizyme Biotechnology Ltd., Liverpool Science Park IC1, 131 Mount Pleasant, Liverpool L3 5TF, UK

4 Novo Nordisk Foundation Centre for Biosustainability, Technical University of Denmark, Building 220, Kemitorvet, 2800 Kongens Lyngby, Denmark

* Correspondence: dbk@liv.ac.uk

\section{check for}

updates

Citation: Shrivastava, A.D.; Swainston, N.; Samanta, S.; Roberts,

I.; Wright Muelas, M.; Kell, D.B. MassGenie: A Transformer-Based Deep Learning Method for Identifying Small Molecules from Their Mass Spectra. Biomolecules 2021, 11, 1793. https://doi.org/10.3390/ biom 11121793

Academic Editors: Lukasz Kurgan and Mark S. Johnson

Received: 24 October 2021

Accepted: 27 November 2021

Published: 30 November 2021

Publisher's Note: MDPI stays neutral with regard to jurisdictional claims in published maps and institutional affiliations.

Copyright: (c) 2021 by the authors. Licensee MDPI, Basel, Switzerland. This article is an open access article distributed under the terms and conditions of the Creative Commons Attribution (CC BY) license (https:/ / creativecommons.org/licenses/by/ $4.0 /)$.
Abstract: The 'inverse problem' of mass spectrometric molecular identification ('given a mass spectrum, calculate/predict the 2D structure of the molecule whence it came') is largely unsolved, and is especially acute in metabolomics where many small molecules remain unidentified. This is largely because the number of experimentally available electrospray mass spectra of small molecules is quite limited. However, the forward problem ('calculate a small molecule's likely fragmentation and hence at least some of its mass spectrum from its structure alone') is much more tractable, because the strengths of different chemical bonds are roughly known. This kind of molecular identification problem may be cast as a language translation problem in which the source language is a list of high-resolution mass spectral peaks and the 'translation' a representation (for instance in SMILES) of the molecule. It is thus suitable for attack using the deep neural networks known as transformers. We here present MassGenie, a method that uses a transformer-based deep neural network, trained on $\sim 6$ million chemical structures with augmented SMILES encoding and their paired molecular fragments as generated in silico, explicitly including the protonated molecular ion. This architecture (containing some 400 million elements) is used to predict the structure of a molecule from the various fragments that may be expected to be observed when some of its bonds are broken. Despite being given essentially no detailed nor explicit rules about molecular fragmentation methods, isotope patterns, rearrangements, neutral losses, and the like, MassGenie learns the effective properties of the mass spectral fragment and valency space, and can generate candidate molecular structures that are very close or identical to those of the 'true' molecules. We also use VAE-Sim, a previously published variational autoencoder, to generate candidate molecules that are 'similar' to the top hit. In addition to using the 'top hits' directly, we can produce a rank order of these by 'round-tripping' candidate molecules and comparing them with the true molecules, where known. As a proof of principle, we confine ourselves to positive electrospray mass spectra from molecules with a molecular mass of 500 Da or lower, including those in the last CASMI challenge (for which the results are known), getting 49/93 (53\%) precisely correct. The transformer method, applied here for the first time to mass spectral interpretation, works extremely effectively both for mass spectra generated in silico and on experimentally obtained mass spectra from pure compounds. It seems to act as a Las Vegas algorithm, in that it either gives the correct answer or simply states that it cannot find one. The ability to create and to 'learn' millions of fragmentation patterns in silico, and therefrom generate candidate structures (that do not have to be in existing libraries) directly, thus opens up entirely the field of de novo small molecule structure prediction from experimental mass spectra.

Keywords: deep learning; electrospray; mass spectrometry; metabolomics; artificial intelligence; generative methods; chemical space; transformers 


\section{Introduction}

The measurement of small molecules within biological matrices, commonly referred to as metabolomics [1,2], is an important part of modern post-genomics. Spectrometric methods are key to their identification. For the deconvolution of matrices such as human serum, chromatography methods coupled to mass spectrometry are pre-eminent $[3,4]$. In mass spectrometry (MS), molecules are ionised and enter a gas phase, commonly using electrospray methods, and the masses and intensities of the fragments-the mass spectrum - contains the diagnostic information that in principle represents a fingerprint for identifying the target molecule of interest. The molecular ion and its fragments may themselves be further fragmented (tandem-MS) using different energies to increase the discriminating power. Identification is currently largely performed by comparing the peaks in the mass spectra obtained with those in a library of mass spectra from known molecules, and the identities may be confirmed by running authentic chemical standards, if available.

The problem with all of the above is that the number of small molecules of potential interest ('chemical space') is vast [5] (maybe $10^{60}[6,7]$ ) whereas the number of synthesised and purchasable molecules as recorded at the ZINC database [8] (http:/ / zinc15.docking.org/) (accessed on 1 November 2021), are just some $10^{9}$ and $6.10^{6}$, respectively (even most of the simplest heterocyclic scaffolds have never been made [9]). Those molecules with available, experimentally determined, high-resolution mass spectra are in the low tens or hundreds of thousands, and even then are highly instrument-dependent. Consequently, the likelihood that any molecule detected in a metabolomics experiment is actually in a library (or even close to a molecule that is [10]) is quite small, and most are not [11-15]. Experimentally, commonly just $10 \%$ of molecules can be identified from what are reproducible spectral features in complex matrices such as serum (e.g., [16-20]), despite the existence of many heuristics such as neutral mass loss, isotope patterns, and so on [21-24]. Solving the mass spectral molecular identification problem is thus seen widely as the key unsolved problem of metabolomics $[4,11,14,15,19,25-40]$. It was also seen as a classical problem in the early development of 'artificial intelligence' [41-44].

It is possible to compute (and hence to generate) all reasonable molecules that obey valence rules and that contain just $\mathrm{C}, \mathrm{H}, \mathrm{O}, \mathrm{N}, \mathrm{S}$ and halogens, which for those with $\mathrm{C}$-atoms up to 17 amount to some 1.66. $10^{11}$ molecules [45]. However, much of the problem of navigating chemical space in search of molecules that might match a given mass spectrum comes from the fact that chemical space is quasi-continuous but molecules are discrete [46]. As part of the revolution in deep learning $[47,48]$, de novo generative methods have come to the fore (e.g., [46,49-59]). These admit the in silico creation of vectors in a high-dimensional 'latent' space ('encoding') and their translation from and into meaningful molecular entities ('decoding'). Although the mapping is not at all simple, small movements in this latent space from a starting point do lead to molecules that are structurally related to those at their starting points $[46,60]$.

By contrast to the 'inverse problem' that we are seeking to solve (where we have a mass spectrum and seek to find the molecule that generated it), the 'forward problem' of mass spectrometry is considerably more tractable (especially for high-energy electron impact mass spectra [61]). Given a known chemical structure, it is possible to fragment the weakest bonds in silico and thereby generate a reasonably accurate 'mass spectrum' of the fragments so generated (e.g., [19,62-71]). Importantly, the advent of high-mass-resolution spectra means that each mass or fragment can be contributed by only a relatively small number of (biologically) feasible [72,73] molecular formulae. Even fewer can come from different, overlapping fragments. Experimentally, we shall assume that the analyst has available a mass spectrum that contains at least 10 fragmentation peaks (probably obtained using different fragmentation energies) including the protonated molecular ion, and we here confine ourselves to analysing positively charged electrospray spectra.

Part of the recent revolution in deep learning $[47,48]$ recognises that in order to 'learn' a domain it is necessary to provide the learning system with far more examples than were historically common, but that the features of (even unlabelled) images [74] or 
text $[75,76]$ can then be learned effectively. Transformers $[77,78]$ are seen as currently the most successful deep learning strategy for 'sequence-to-sequence'-type problems such as language translation [79-81]. Obviously SMILES strings constitute a language and while it may be less obvious that mass spectra do so, they too can be seen to represent a sequence of elements, in this case high-resolution peaks. The 'spectrum-to-structure' problem may thus be cast as a language translation problem. In particular, much as how human infants learn from their surroundings, it has been established in the transformer type of architecture [77] that the initial 'pre-training' can if necessary be carried out in an entirely unsupervised manner, with 'fine-tuning' being sufficient to learn the domain of interest $[75,78,79,82,83]$. We recognise that this is also possible for the space of small molecules, where the features of interest are their (mass spectral) fragments. However, in this case we may in fact generate matched pairs of spectra and molecular structures from the encoding (as their SMILES strings) of the relevant molecules, and treat this as a supervised learning problem.

We thus considered that one might fruitfully combine these two main elements (molecular generation and molecular fragmentation) with an objective function based on the closeness of the predicted mass spectrum to the experimental one that one is trying to fit. This would allow us to 'navigate chemical space intelligently' [56] so as to provide the user with a set of molecules (ideally one) that generates something close to the observed mass spectrum and hence allows identification of a candidate molecule, even if such a molecule does not appear in a curated database. We here implement this approach, that we refer to as MassGenie, for learning positive electrospray ionisation mass spectra as input and the molecules that might generate them (as output). MassGenie offers an entirely novel and very promising approach and solution to the problem of small molecule identification from mass spectra in metabolomics and related fields. We believe that it represents the first use of transformers in molecular identification from (mass) spectra. A preprint (dated 26 June 2021) has been lodged [84].

\section{Materials and Methods}

\subsection{Small Molecule Datasets Used}

We first create and combine two datasets, that we refer to as ZINC 6M and ZINC 56M. The ZINC 6M dataset originally was a mixture of 1381 marketed drugs [85], 158,809 natural products [86], 1112 recon2 metabolites [85], 150 fluorophores, and molecules from a subset of the ZINC15 database (subset1 contained 2,494,455 and subset2 ("2D-cleandrug-like-valid-instock") contained 6,202,415 molecules. After canonicalization of the SMILES and the removal of duplicates we ended with 4,774,254 unique SMILES. We filtered any duplicates as well as structures for which the $m / z$ of the protonated molecular ion exceeds 500. This is because comparatively few molecules had peak (mass) values higher than 500 and including them would have led to a drastic increase in the input dimensionality of our model, constraining the adjustment of other hyperparameters such as batch size, model size, etc away from their optimal values. From this, we obtained $\sim 4.7 \mathrm{M}$ molecules encoded as SMILES (Table 1). Despite these large numbers, some atoms are under-represented. For instance, we did not feel that there were sufficient S-containing molecules to give us confidence with the experimental mass spectra, although the system performed well with those generated in silico.

Table 1. Summary of datasets.

\begin{tabular}{|c|c|c|c|c|c|c|c|}
\hline Data Source & Recon 2 & $\begin{array}{c}\text { Natural } \\
\text { Products }\end{array}$ & $\begin{array}{l}\text { Marketed } \\
\text { Drugs }\end{array}$ & FLUOROPHORES & $\begin{array}{l}\text { ZINC15- } \\
\text { subset1 }\end{array}$ & $\begin{array}{l}\text { ZINC15- } \\
\text { subset2 }\end{array}$ & Total \\
\hline $\begin{array}{c}\text { Original } \\
\text { smiles count }\end{array}$ & 1112 & 158,809 & 1381 & 143 & 249,455 & $6,202,415$ & $6,613,315$ \\
\hline $\begin{array}{l}\text { Canonical } \\
\text { smiles count }\end{array}$ & 1056 & 148,971 & 1357 & 115 & 247,804 & $4,425,891$ & $\begin{array}{c}4,825,194 \\
(4,774,258- \\
\text { unique) }\end{array}$ \\
\hline
\end{tabular}


For ZINC 56M, we randomly sampled 56M SMILES from a set of $\sim 794 \mathrm{M}$ within ZINC15 tagged as "2D-clean-druglike-annotated". After canonicalization and duplicate removal, we obtained $\sim 53 \mathrm{M}$ SMILES and again from these sampled randomly $\sim 3.2 \mathrm{M}$ structures and applied the same procedure. In this purposely chosen subset of ZINC, the number of molecules with molecular ion peak values more than 500 were much lower $(21,454)$; there were also far fewer duplicates. We then combine both datasets. The combined dataset thus had 7.9M SMILES strings augmented to be in triplicate, of which 100,000 were reserved as a validation set (these happened to include cytosine, see later).

In addition, for fine-tuning, we made use of 201,858 molecules from the GNPS dataset (obtained from https://gnps-external.ucsd.edu/gnpslibrary/ALL_GNPS.json) (accessed on 1 November 2021). The dataset originally had 439,996 data samples. We then filtered the dataset for fine tuning based on the following: first, we drop the rows containing "N/A" values. Then we filter out the samples that do not have "positive" ion mode. This left us with 271,497 samples in the dataset. After this, we applied each of the three filters that we applied in the training set, i.e., the removal of samples with $m / z$ values exceeding 500, of samples with SMILES string length more than 99, and of the samples where the number of $m / z$ peaks is more than 100 . After this, we finally obtain a refined trainable dataset of 201,858 molecules. However, not all of these 201,858 molecules were used for training as 10,000 samples were held out as another potential test set.

We also produced a second completely independent test set of over 1000 separate molecules from ZINC 56M, fragmented as below, that were tested single-blind. Finally, we utilised experimental mass spectra generated as part of our current metabolomics studies $[18,87]$ and in the CASMI competition.

\subsection{In Silico Fragmentation Method}

To fragment molecules in silico, a bespoke implementation of MetFrag [64,65] was developed, allowing for the generation of theoretical fragmentation spectra which were tailored to approximate those found in experimental mass spectra libraries. Like MetFrag, our implementation (FragGenie, available at https://github.com/neilswainston/FragGenie) (accessed on 1 November 2021) utilises the Chemistry Development Kit [88] to represent small molecules as a matrix of atoms and bonds. Each bond is broken serially with each bond break resulting in either two fragments, or a single fragment in the case of ring-opening breaks. This process continues recursively, with each fragment being subsequently fragmented further until a specified recursion depth or a minimum fragment mass is achieved. A number of adduct groups may then be applied (e.g., protonation and sodiation) with the resulting collection of fragment $m / z$ values providing a theoretical fragmentation spectrum for a given small molecule. (In addition, and largely for the purposes of debugging, chemical formulae and a list of bonds broken may also be generated for each individual fragment.) Such a naïve approach of breaking all bonds recursively quickly generates an excessive number of potential fragments. As such, the method was tuned against existing fragmentation spectra in the MoNA database (https: / / mona.fiehnlab.ucdavis.edu) (accessed on 1 November 2021) in order to limit the nature of bond breaks to those which are most often found in measured spectra. While the fragmentation method of FragGenie can be filtered to include or exclude any specified bond type, for this work fragmentation was limited to single, non-aromatic bonds, protonated adducts, and a fragmentation recursion depth of 3 .

Note that throughout we are in effect using only individual (not tandem) mass spectra, though these may be constructed in silico from experimental data by combining mass spectra generated at different fragmentation energies (e.g., by changing cone voltages in an electrospray instrument or by varying the pressure of collision gases). We also assumed that 'real' (experimental) mass spectra would contain a known, protonated molecular ion (reflecting a positive electrospray experiment) of a mass of sufficient accuracy to provide a molecular formula. Thus, we do not directly implement tandem spectra: 'fragments of fragments' are seen simply as having the molecular ion of the largest peak and may be 
treated accordingly in a similar manner to that described for the original molecule. This strategy necessarily misses fragments that undergo rearrangements and unknown mass losses, etc.

The fragmentation method used in our program does not seek to predict intensities directly, but merely the presence or absence of a peak with a certain high-resolution mass $(m / z)$. We next apply a novel data augmentation procedure to make the model learn to prioritise the input peaks, to encourage the learning of peaks whose presence tended more strongly to suggest the corresponding SMILES. We observe from the MetFrag algorithm that certain types of bonds are broken in the first step. These are the ones that correspond to the highest intensity when compared with the peak list obtained from a real mass spectrometer. Also, these peaks have the same mass accuracy both theoretically as well as in the real mass spectrometer. Intuitively, this list of peaks always has (and is taken to have) the molecular ion. Subsequently, in the 2nd step, peaks with comparatively lower ranges of intensity are obtained and also with slightly higher noise in them when compared with the real peaks from the mass spectrometer. The pattern is repeated once more.

Thus, for each SMILES molecule, we generate three data samples or molecule peak pairs, one for each step of bond breaking up to three. This technically leads to a 3-fold increase in our overall data. However, in our experiments we only consider samples with a maximum input length of 100. Therefore, any SMILES molecule-FragGenie peaks pair, where the length of the list of peaks is more than 100, is dropped from the dataset. This leaves us with the 21 M SMILES that we use for training our model.

Throughout we assume that we have high-resolution mass spectral data with a precision of better than 5ppm, as is nowadays common (and is easily achieved in our own Orbitrap instruments [18]). The present paper utilises only ES+ data. For the input mass spectral data we applied a binning procedure. Data were filtered such that the peaks have an upper bound of $m / z$ 500. This effectively produces a fixed precision of 0.01 . Even for a mass of 500 , a precision of 5 ppm equates to a $\Delta$ mass of only 0.0025 , so these bins are adequate.

Thus, we consider the binning range from 0 to 50,000 and to categorize the peak into these peaks we multiply the peaks by 100 so what we get is essentially its bin number. Afterwards, the number of bins is projected as the dimensionality of the input, and the category value for every peak which we get from carrying out the above procedure reflects the index which is set in its input vector.

The input data consist of the list of values of peaks in floating point numbers. We constrained the number of peaks to be less than or equal to 100 . Note that we do not use abundance data, because they depend entirely on the strength of the collisions; instead, we assume a series of experiments using collisions (or other molecular deconstruction near the source) sufficient to provide at least 10 peaks from the molecule of interest.

For the output data, we first split the SMILES into a list of individual atom types (C, N, O, P, etc.) and other characters such as "[", "]", " (", " $)^{\prime \prime}$, "=", " " , etc. The total vocabulary size was 69 including the 4 special tokens <sos> (start of sequence), <eos> (end of sequence), <unk $>$ (unknown), and $<$ pad $>$ (padding) and this defines the output dimensionality of our transformer model as now described fully.

\subsection{The Transformer Model}

We here used the standard transformer model [77] and cast the problem as a peaks-toSMILES translation problem (Figure 1A). Our transformer encoder, written in PyTorch, is composed of 12 transformer encoder layers plus 12 transformer decoder layers. The output dimensionality of all the sub-layers, as well as the embedding layers in both encoder and decoder, was set to 1024. Besides this, each Multi-Head attention layer of our transformer model was formed of 16 attention heads in total. We used dropout [89] heavily; its value for the complete transformer model was set to $20 \%$. Lastly, the dimensionality of the feed-forward layer present inside all of the transformer encoder and decoder layers, and applied to every position individually, was set at 4096. Effectively, we allow the model to 
see the entire input sequence containing peaks and impose a target attention mask in the decoder stack to prevent it from being exposed to the subsequent positions. Altogether, our model consisted of $\sim 400 \mathrm{M}$ trainable parameters.

A

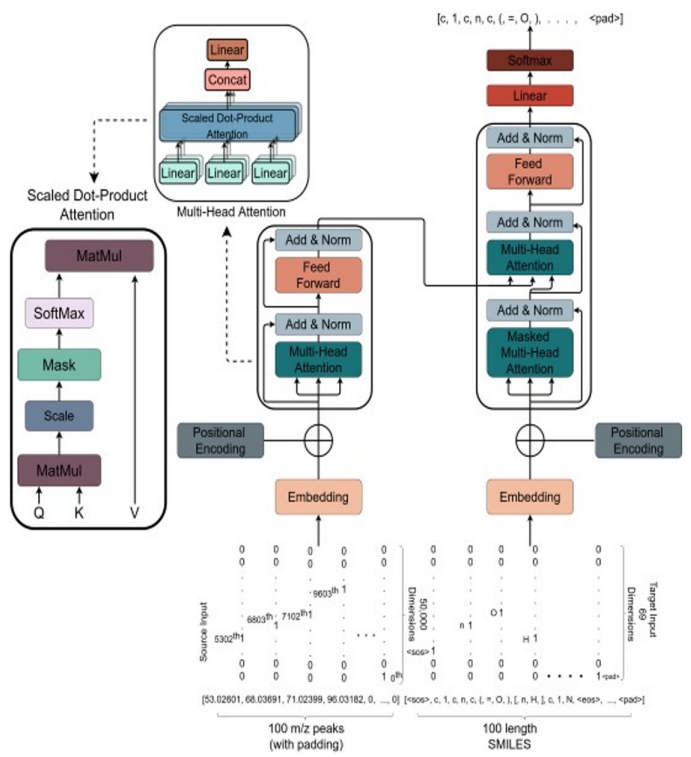

B

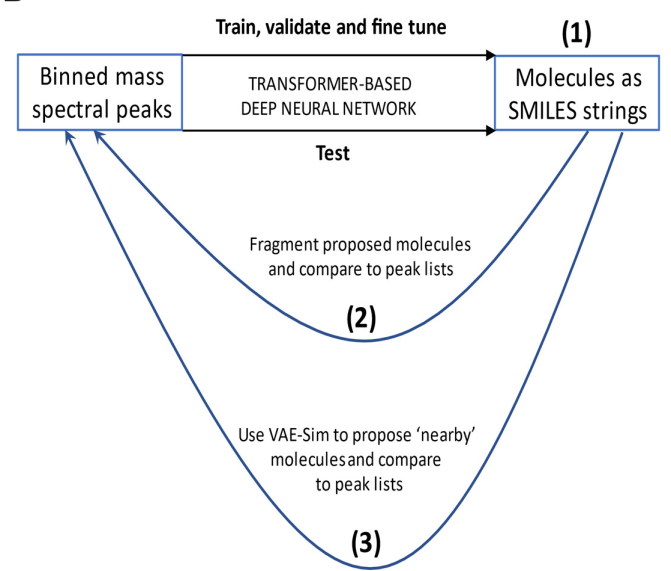

C

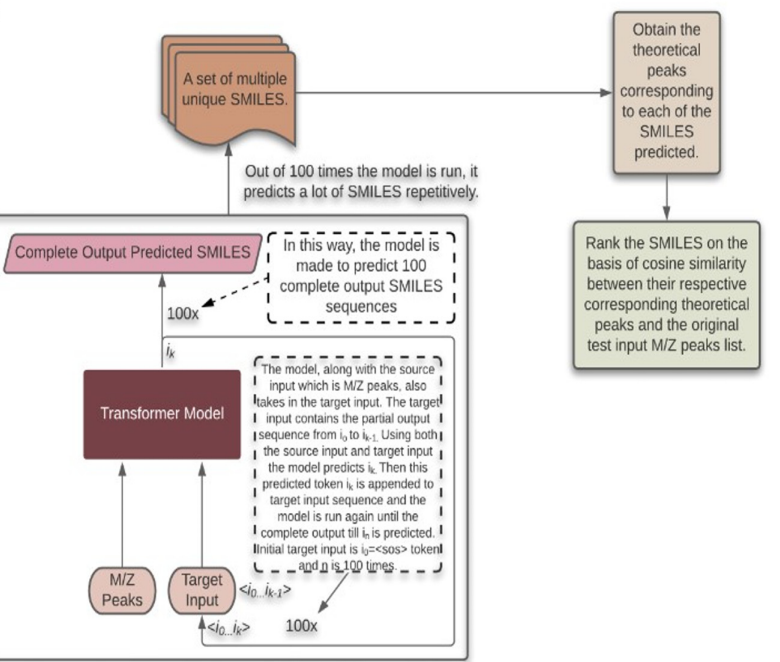

Figure 1. Cont. 
D

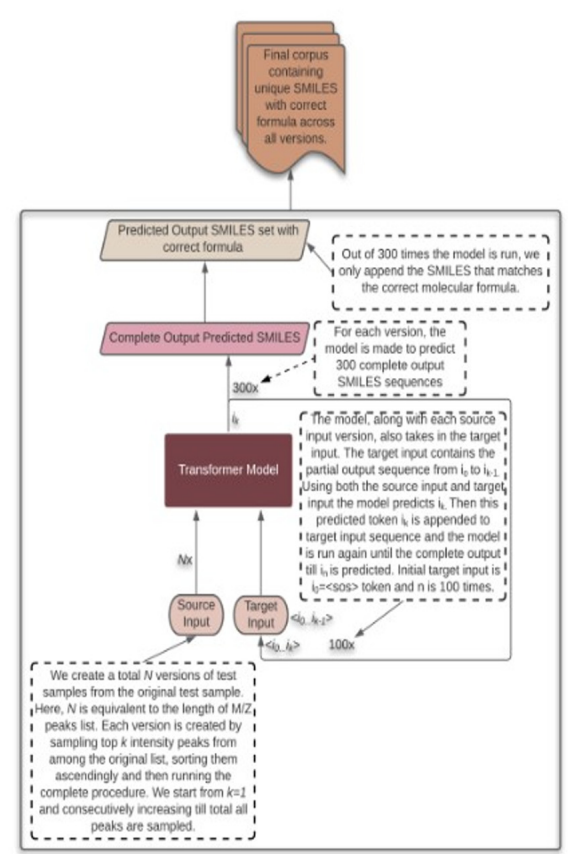

Figure 1. The overall strategy behind MassGenie. (A). The overall architecture and structure of the transformer used in MassGenie, our deep learning system for identifying molecules from their mass fragments (spectra). (B). The three basic strategies available to us for relating mass spectral peak lists to the molecules whence they might have come. (1) The transformer outputs only a single molecule. (2) We can take a series of candidate molecules, generate candidate mass spectra in silico using FragGenie, and compare them with the experimental mass spectra, using cosine similarity to rank order the candidates. (3) We can use VAE-Sim to generate further candidate molecules that are 'close' in chemical space (and possess the correct molecular formula) and rank those as in (2). This is done for both FragGenie-generated spectra (C) and experimental mass spectra (D).

As indicated, we use the transformer model, which is originally a language translation model, to 'translate' MS2 peak lists into SMILES. When used for language translation problems, and when given an input sentence, such a model, tends to predict multiple sentences that could be the corresponding translation of the input sentence. Some of the predicted candidate sentences even have a very similar meaning. Hence, when we apply the transformer model to our problem, we evoke a similar kind of behaviour (albeit in a different domain or context): our model predicts different SMILES strings each time when we run the model on the particular list of peaks. All of these are considered to be the candidate predictions of the molecules. Now, from our experiments and observations, what we have witnessed is when the model is pretty 'confident' about its predictions, it does predict the same output multiple times. However, the unique predictions are considerably smaller than the number of times it was made to predict. Also, there is typically a very high similarity between the candidate predictions. By contrast, when the model is unsure or cannot decipher the patterns properly, the model gives a set of unique predictions that is almost equal in size to the number of times the model was run for predictions. Also, the candidate predictions tend to have a considerable amount of dissimilarity between them. This admits, qualitatively, a measure of the certainty in the prediction from a transformer model. We also use the information about whether the predictions have the correct molecular formula.

\subsection{Training Settings}

We first trained a model with in silico fragments only. By applying the data augmentation described above, we used a total of $\sim 21 \mathrm{M}$ data samples, from which we reserve 100,000 samples for validation and utilize the rest for training. The data were batched as 896 samples per batch. We adopt the same optimizer setup as implemented in the original 
paper [77], namely the Adam optimizer with $\beta 1=0.9, \beta 2=0.98$ and $\varepsilon=10^{-9}$, but with the following slight changes. First, we double the warmup steps to 8000 . Secondly, we apply scaling (as in [89]), to the overall learning rate, dependent upon the batch size considered for training. We use the open-source PyTorch library to construct and train the complete model for the problem. We trained our model on an NVIDIA DGX $8 \times$ A100 system; this required approximately one day of training to reach its best validation loss. This is about 30-fold quicker than a 4-GPU (V100) machine that we have used previously [60] for a different transformer-based deep learning problem.

\subsection{Testing the Results}

As with any mass spectral prediction program, the user anticipates being presented with a list of candidate predicted molecules, if possible in a rank order, and this is what we supply. It is then, of course, up to the user to validate the predictions, most persuasively [90] by running the standard under the same conditions that generated the original mass spectrum. We employ two procedures to analyse the performance achieved by the trained model. The first procedure ("transformer search") uses solely the transformer. When queried with a testing input list of peaks, we run the model 100 times to predict a list of output SMILES from the list, and filter this to produce a non-redundant set of predicted SMILES, also in some cases filtering to remove any molecules with the incorrect molecular formula (from the predicted molecular ion). We also have access to round tripping procedures, using our fragmentation system to regenerate the theoretical list of MS peaks corresponding to each of the non-redundant predicted SMILES. This allows us to compare the SMILES based on the similarity between the theoretically generated list of peaks and the original query list of testing peaks.

In a second procedure ("VAE search"), we use predictions from the transformer to search the local latent space in an updated version of our trained variational autoencoder [60]. We take the top-1 predicted molecule and produce a new molecule by moving a small Euclidean distance away. This is done by taking randomly 10,000 samples within a annular fixed radius from the predicted molecule's position in the 100-dimensional latent space [60]. We also varied the radius 50 times with a fixed size of $10^{-2}$. This can generate many molecules beyond those on which the variational autoencoder was trained [46,60]. Out of those 500,000 samples typically $\sim 0.1 \%$ are valid samples (due to the effectively infinite size of the VAE latent space compared to the finite number of locally valid molecules). This takes approximately $4 \mathrm{~min}$, whereupon a set of molecules with the correct mass ion is chosen.

Experimental mass spectra were generated from pooled human serum or from chemical standards using an Orbitrap mass spectrometer and the methods described in $[18,87]$ with standard settings, and were visualised using Compound Discoverer (Thermo).

Testing our system using 'real' (experimental) mass spectral peaks is somewhat different from testing it on the peaks generated in silico, for a number of reasons. First, we do not know exactly which peaks are more 'important' or even which may be true peaks, and which are erroneous due to contaminants. However, empirically, we do recognise that the higher intensity mass peaks tend more usually to be correct ones; as the intensity decreases, the noise in the peaks starts to increase and they eventually become erroneous (i.e., they do not come from the target molecule of interest). Based on this, we initiate a variable $k$, firstly with an initial value of 1 . Then, we sample the top $k$ intensity peaks. We sort them so that the peaks are always in an ascending order of $m / z$. We run the model for $\mathrm{N}=300$ times and obtain a list of predictions. Since we already know the molecular formula from the exact mass of the molecular ion, the predicted list of SMILES molecules is filtered as required to match the known chemical formula. We do multiple iterations of this process, increasing the value of $\mathrm{k}$ by one. The number of iterations equals the total number of peaks in the test sample. Finally, we output the final list of candidate predictions. Because of the relative paucity of S- and P-containing molecules in our training set, we confined our analysis of experimental spectra to those containing solely $\mathrm{C}, \mathrm{H}, \mathrm{O}$ and $\mathrm{N}$. 


\subsection{Testing with CASMI Data}

We obtained the dataset for the 2017 CASMI challenge from http:/ / casmi-contest.org/ 2017 /index.shtml (accessed on 1 November 2021). In total the dataset contained peaks for 243 challenges. We apply the same preprocessing and filtering that we applied for GNPS and ZINC datasets. Thus, we need to filter the examples based on several constraints. First, we remove all the challenges which have mass spectra in "NEGATIVE" ion-mode and only keep the "POSITIVE" ion-mode, since our model was trained on all positive mass spectra. Further, we remove all the mass spectra whose $m / z$ values of any peak goes greater than or equal to 500. Next, we also remove all the challenges whose mass spec length is equal to or greater than 100. And we also do the same with SMILES. Finally, we are left with 93 challenges (out of the initial 243 challenges) on which we test our model.

We also follow a similar testing procedure as for GNPS. For each challenge, we first run a for loop to sample the top-K intensity peaks, where $\mathrm{K}$ goes from 1 to $\mathrm{n}(\mathrm{n}=$ total no. of peaks). Once we have those top-K intensities, we sort them based on the $m / z$ values and feed them to the MassGenie model. With that $m / z$ intensity value list as an input, we make MassGenie predict 100 times. On the list of 100 predictions, we apply a filter to remove SMILES strings that do not have a correct chemical formula. So this leaves us with SMILES strings with the correct chemical formula. We form a set of these remaining SMILES strings to remove any repeated outputs. Hence, this forms our prediction output set.

Once we have the solution set, we estimate the Tanimoto similarity between the correct SMILES string and each of the SMILES strings in the prediction set and consider the prediction for which the Tanimoto comes out to be the highest. The Tanimoto similarity is estimated using RDKit's RDKFingerprint method.

\section{Results}

The first task was to train the deep transformer network described in Methods with paired in silico mass spectra as inputs and molecular structures as outputs. The network so generated (the code will be made available in due course) had some $400 \mathrm{M}$ nodes, and was trained over a period of $\sim 1$ day (actually $19 \mathrm{~h}$ ). We used a validation set of 100,000 molecules and use the performance (validation loss) achieved on that set as a metric to ascertain that we use the best version of the trained model for the testing. After every epoch, we run the model on the validation set and record the performance (validation loss) on it. And if the best validation loss so far is found, we record the weights of the model for that epoch. We repeat this process after every epoch until a significant number of epochs have passed without seeing any improved validation loss. The transformer architecture is given in Figure 1A, while the overall procedure including the two 'round tripping' strategies is given in Figure 1B. Figure 1C,D illustrate the strategy as applied to FragGenie-generated peaks and to experimental mass spectra.

By way of example, the following peak list was generated by FragGenie from the molecule shown on the left of Figure 2, while the candidate predictions are given on the right-hand side of that figure.

$[60.02059,66.02125,70.00248,81.03214,81.04474,96.05563,109.99178,112.04313,116.018036,125.002686,127.05403$, $127.91178,131.02895,131.04152,134.03624,135.01643,137.9867,146.05243,149.04715,150.02734,150.03992,152.9976$ 153.98161, 165.05083, 168.99251, 201.92743, 205.04015, 216.93832, 229.0846, 229.92235, 233.03505, 236.89629,

239.06648, 244.93324, 245.91727, 249.02995, 251.90718, 255.03694, 259.01187, 260.9282, 264.8912, 267.0614, 274.03534, 279.08142, 279.9021, 283.03186, 283.05634, 287.00677, 295.05185, 296.9758, 299.02676, 302.03024, 303.00168, 316.92114, 324.97076, 331.94464, 340.96567, 340.9657, 344.9161, 346.97266, 350.94757, 359.9396, 360.91104, 362.94308, 365.97107, $366.918,374.9676,378.9425,381.9415,385.9164,386.98758,390.93802,390.9625,390.96252,393.966,394.91293$, $394.93744,400.9399,402.958,406.93292,406.93295,409.93643,410.90787,413.91135,428.93484,444.92978,459.95325]$ 

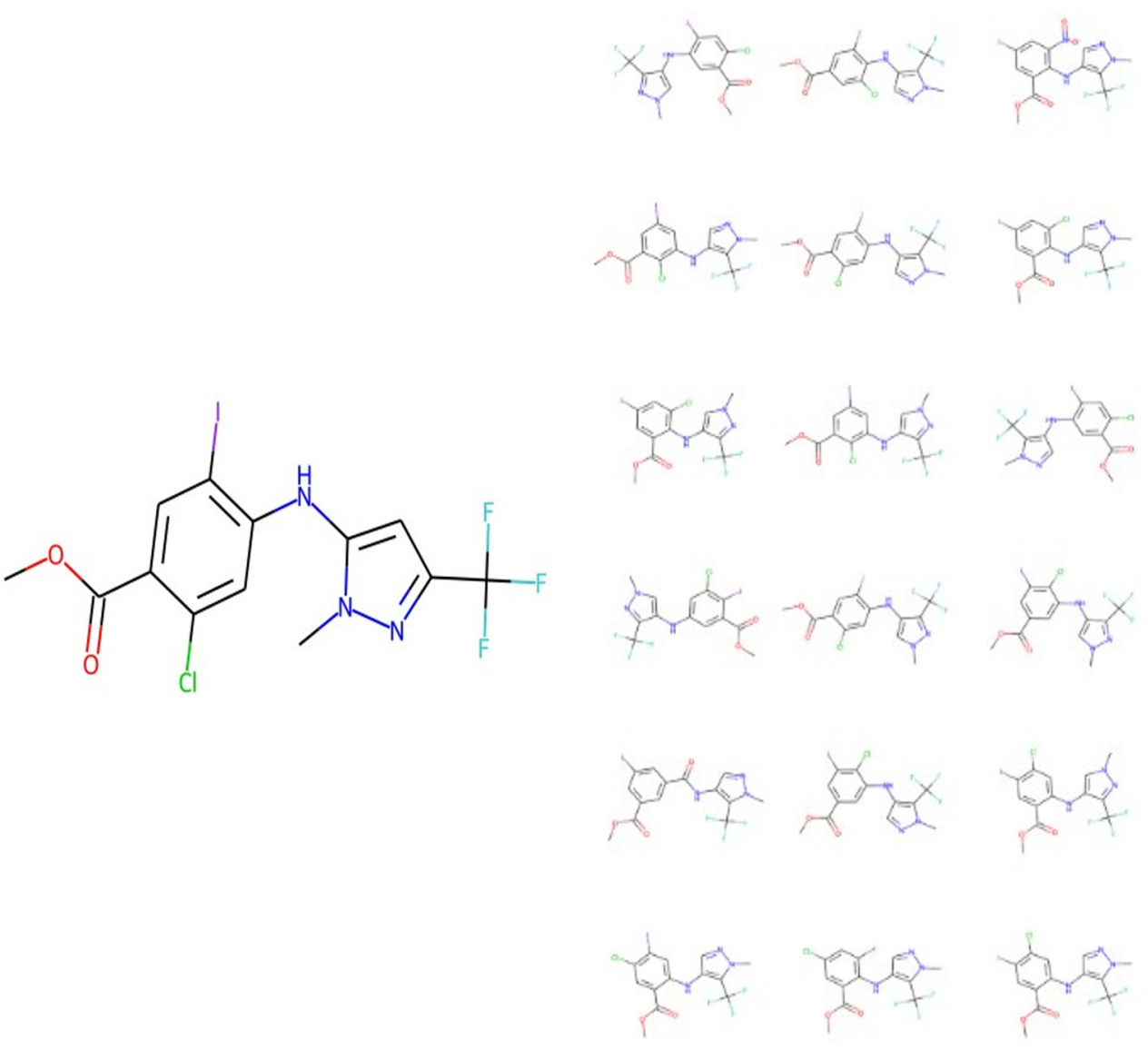

Figure 2. Illustration of the means by which MassGenie can predict a series of candidate molecules. In this case, the 'true' molecule is shown on the left, and 18 candidate molecules (from 100 runs) shown on the right. It is clear that all are close isomers, containing a methoxybenzoate moiety linked via a secondary amine to a pyrazole ring with a trifluoromethyl substituent.

As seen (Figure 2), while the model was run a hundred times, it predicted only 18 unique molecules, and each of them is a very close isomer. The closest one has a Tanimoto similarity of 0.95 to the true molecule when encoded with the TYPICAL fingerprint strategy [86] as described next.

We ran a test set of 1350 separate molecules through the network using a single-blind strategy (mass peak lists of molecules not in the training or validation sets were provided by SS and were then tested by ADS). Each prediction took about $2.6 \mathrm{~s}$ on our A100 system, which when done $100 \times$ meant $\sim 4 \mathrm{~min}$, or $98 \mathrm{~h}$ for the full 1350 . The concept of molecular similarity is quite elusive [91,92], and the usual means of assessing it (an encoding of properties or molecular fingerprints followed by a similarity metric such as that of Jaccard or Tanimoto [93]) depends strongly on the encoding [86], and is to some degree in the eye of the beholder. These comparisons are normally determined pairwise, though the parametrisation can be done using large cohorts of molecules [60]. For present purposes, we estimated similarity on the basis of what we refer to as the TYPICAL similarity [86]; this uses a set of encodings from rdkit (www.rdkit.org) (accessed on 1 November 2021) (we used rdkit, atom pair, topological torsion, MACCS, Morgan and Pattern) followed by the Jaccard metric, and takes the values of whichever returns the largest (this was almost invariably the Pattern encoding).

Armed with the network's estimates of the molecular structures and the then-disclosed identities, we used the above TYPICAL strategy [86] to assess the Tanimoto similarity (TS) between the network's best estimate and the true molecule. Of these, $1117(82.7 \%)$ were precisely correct, 1338 had a TS exceeding $0.9(99.1 \%)$ and only $2(0.15 \%)$ had values of TS below 0.8. The data are given in Supplementary Table S1, and the structural closeness 
of some of the molecules with a TS of 0.9 is indicated in Figure 3. It is clear that a TS of greater than 0.9 does implies a molecule very close in structure to the correct molecule. Those shown give a clear indication of both the difficulty and the success of the task as they are indeed significantly complex structures. (Note that in this case 233 of the molecules contained S, of which 203 (91\%) were nonetheless divined correctly.)

\section{Analysis of 1350 in silico mass spectra}

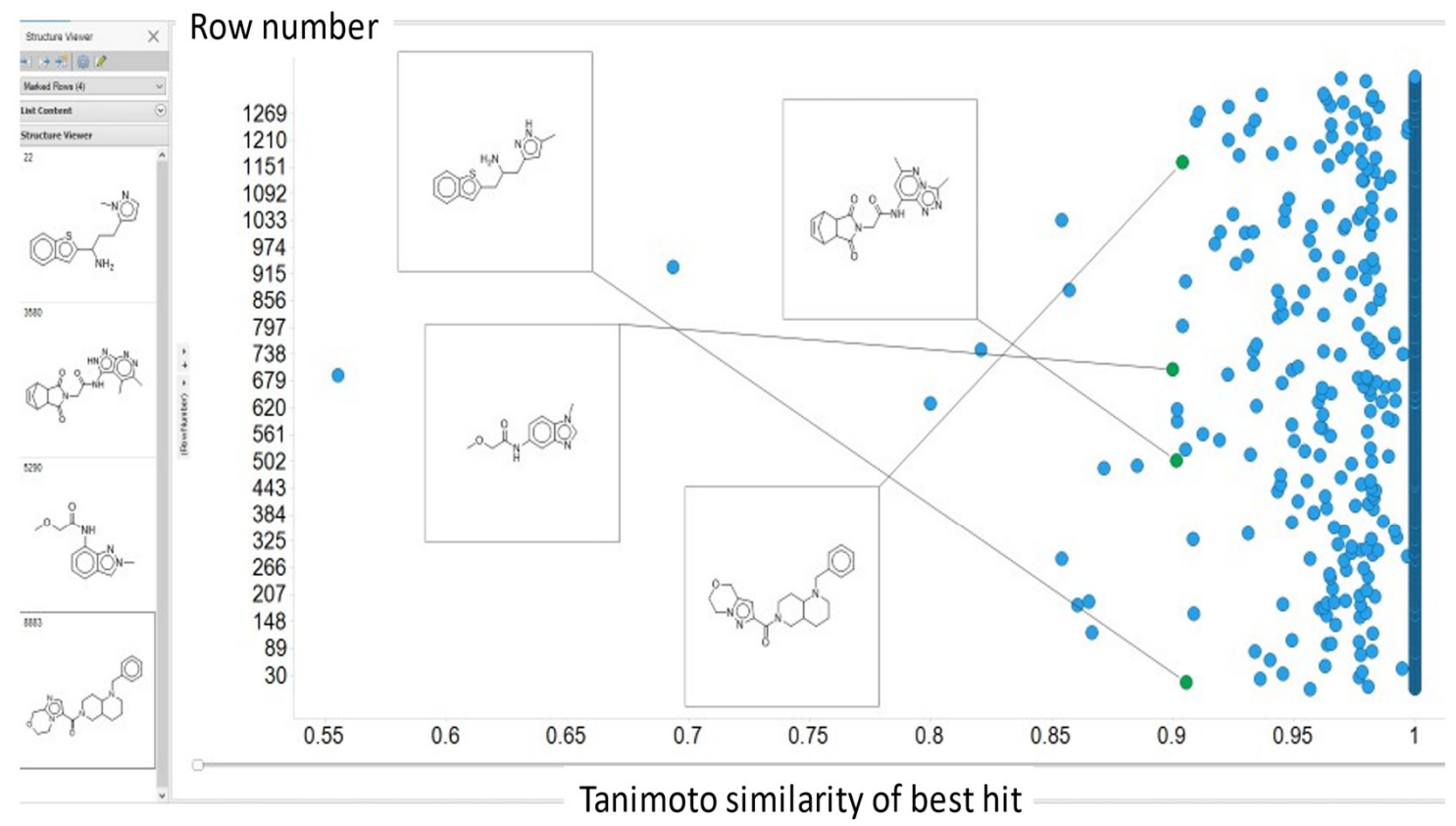

Figure 3. Analysis of a test set of predictions of the molecules behind 1350 in silico-fragmented mass spectral peaks. The peak lists were passed through the transformer after it had been trained and fine-tuned as described in methods. The analysis was done single-blind and the results fed back. The 'closeness' between the molecule estimated and the true molecule is given as the highest Tanimoto similarity based on six encodings. Four estimated molecules with a TS of $\sim 0.9$ are illustrated, together (at left) with the 'true' molecules. It is again obvious that they are extremely close structurally.

In a few cases, MassGenie failed to produce a molecule with the correct molecular formula. In this case, we were able to encode its best estimates in the latent space described in our VAE-Sim paper [60], and move around the latent space until we found the closest molecule with the correct molecular formula. We ran 50 of these. Figure 4 illustrates the results for 10 molecules from those whose TS in Figure 3 was between 0.9 and 0.95, raising the average TS from 0.92 to 0.96 , and producing structures that were clearly closer to the true one. The VAE-Sim strategy is thus a very useful adjunct to the direct Transformer approach.

Figure 5A further illustrates the use of VAE-Sim in cases where there is a mass difference between the known molecular ion and MassGenie's predictions, both in terms of the rank order of cases where the mass difference is smallest (orange) or the Tanimoto similarity is greatest (blue). Figure 5B illustrates three examples in which VAE-Sim can effectively 'recover' the correct molecule by searching locally to the transformer's top hits that just have the wrong mass. 


\section{Use of VAE-Sim for exploring local candidate structures}
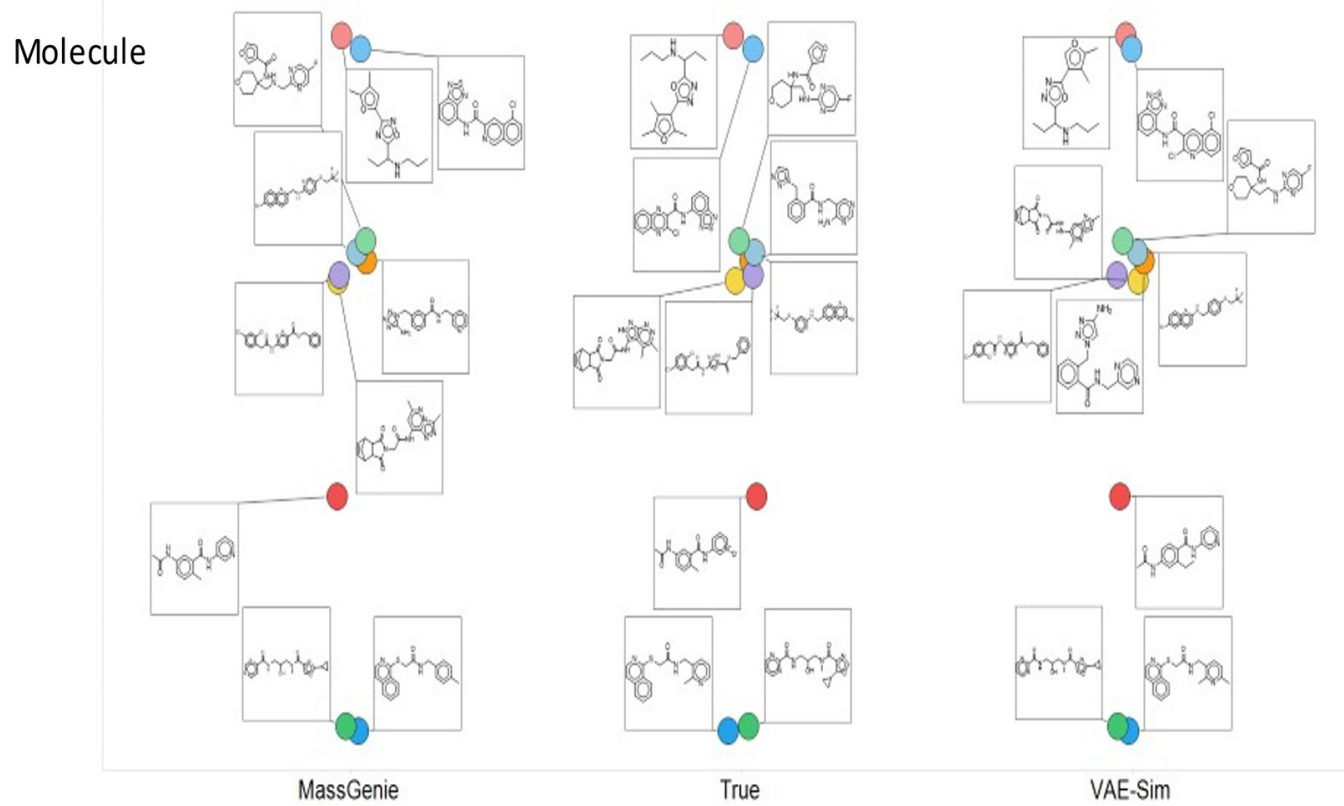

Figure 4. Local candidate structures generated by VAE-Sim. Based on 10 molecules taken from Figure 3, where the Tanimoto similarity between the best hit and the true molecule was in the range 0.9-0.95. VAE-Sim increases the number of candidates, in many cases improving their closeness to the true molecule.

The number of fragments typically generated by FragGenie commonly exceeds those available in real electrospray mass spectra such as those available via MoNA $[23,94]$ or GNPS $[23,94]$ (and the experimental spectra are not at all identical in different databases). This said, generative deep learning methods of this type are sometimes capable of reconstructing whole entities (words or images) from partial, heavily masked inputs [95], so we considered that it might be reasonable that a transformer trained solely on in silico fragments might nevertheless be able to make predictions from experimentally obtained mass spectra that contained many fewer. We first used both purified standards of known structure and molecules assessed during experimental LC-MS(-MS) runs where spurious peaks can appear because of imperfect separations.

Our present LC-MS/MS method of the pooled human serum used [18], based on that in [3], shows 3009/3478 reproducible metabolites in $\mathrm{ES}^{+} / \mathrm{ES}^{-}$mode, respectively, that appear in at least $50 \%$ of replicates and for which we have tandem mass spectra. Of these only 391/213 have confirmed entries in the mzcloud library (https:/ /www.mzcloud.org/) (accessed on 1 November 2021). Since the space of known and purchasable molecules far exceeds those for which we have experimental mass spectra, it is possible to assess those for which the proposed spectra are of purchasable compounds. It could thus be observed that experimental spectra of known compounds in serum often contained many spurious peaks not present in the standards. Consequently, we also used known standards that were run experimentally. 


\section{A}

\section{MassGenie performance}

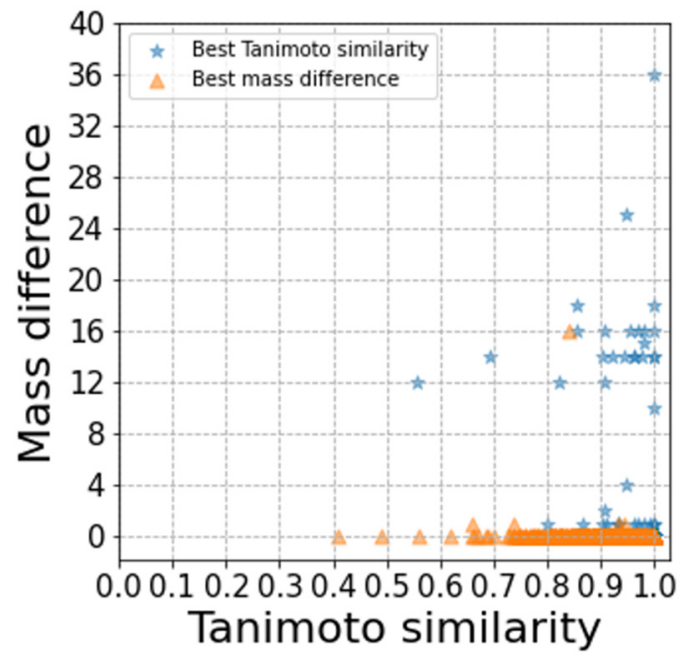

B
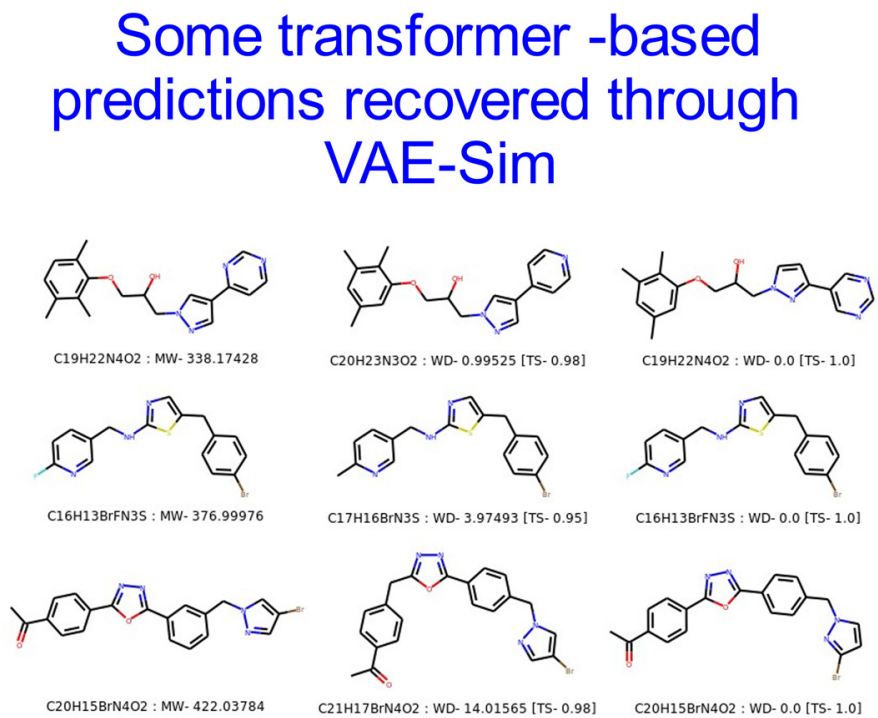

$20 \mathrm{H} 15 \mathrm{BrN} 4 \mathrm{O} 2:$ MW- 422.0378

C21H17BrN4O2 : WD- 14.01565 [TS- 0.98]

MassGenie

After VAE -Sim

Figure 5. Local search where mass predictions are inaccurate. (A). All examples from Figure 3 in which the transformer predicted molecules with slightly incorrect masses. VAE-Sim was used to search locally and generate further candidate structures that were round-tripped using FragGenie to produce mass spectra that could again be ranked in terms of TS to the known, true molecule given either the optimal mass difference or the optimal TS. (B). Three examples showing the ground truth, the transformer's best estimate, and the best (and accurate) prediction after the candidate pool of molecules was enhanced using VAE-Sim.

This experiment was again done single-blind, by which IR and MWM provided peak lists and knowledge of the molecular ions for molecules whose structures they had confirmed by running authentic standards or by other means. These are shown in Table 2, along with the performance of MassGenie. As with the interpretation of FragGenie peak lists, we obtained multiple candidates from each analysis. In practice, many of them possessed different SMILES representations but were actually of the same molecule. We therefore converted all the SMILES variants into a single canonical SMILES with its structure and assessed the most common. Figure 6 gives an example. As with FragGenie, 
the table thus uses as its top candidate the structures that were most frequently proposed. In some cases, MassGenie did not propose a structure with the correct mass ion.

Table 2. Predictions by MassGenie of molecular structures from experimental positive electrospray mass spectra, Molecules were provided single-blind to MassGenie and the ranked outputs are recorded. 11 of the 16 molecules could be predicted. Colors reflect the precision of the results as described in the Result column.

\begin{tabular}{|c|c|c|c|c|c|c|c|c|}
\hline Compound & $\begin{array}{c}\text { Formula } \\
\text { (Predicted } \\
\text { by MS } \\
\text { Software } \\
\text { and Correct } \\
\text { by Standard) }\end{array}$ & $\begin{array}{c}\text { Monoisotopic } \\
\text { Mass }\end{array}$ & $\mathbf{M}+\mathbf{H}$ & Matrix & $\begin{array}{l}\text { Number } \\
\text { of Peaks/ } \\
\text { Frag- } \\
\text { ments }\end{array}$ & $\begin{array}{c}\text { SMILES } \\
\text { Canonical No Isotopic or } \\
\text { Chiral Markings }\end{array}$ & Result & Comment \\
\hline $\begin{array}{l}\text { Lauryl-1- } \\
\text { carnitine }\end{array}$ & C19H37NO4 & 343.2723 & 344.2801 & $\begin{array}{l}\text { STD in } \\
\text { water }\end{array}$ & 11 & $\begin{array}{c}\operatorname{cccccccccccc}(=\mathrm{O}) \mathrm{OC}(\mathrm{C} \\
[\mathrm{N}+](\mathrm{C})(\mathrm{C}) \mathrm{C}) \mathrm{CC}(=\mathrm{O})[\mathrm{O}-]\end{array}$ & $\begin{array}{l}\text { Part of } \\
\text { options }\end{array}$ & $\begin{array}{l}\text { Part of the } \\
\text { options (3 } \\
\text { out of 28) in } \\
\text { ChemSpider }\end{array}$ \\
\hline $\begin{array}{l}\text { Stearoyl-1- } \\
\text { carnitine }\end{array}$ & $\mathrm{C} 25 \mathrm{H} 49 \mathrm{NO} 4$ & 427.3662 & 428.374 & $\begin{array}{l}\text { STD in } \\
\text { water }\end{array}$ & 7 & $\begin{array}{c}\text { CCCCCCCCCCCCCCCCCC } \\
(=\mathrm{O}) \mathrm{OC}(\mathrm{C}[\mathrm{N}+](\mathrm{C}) \\
(\mathrm{C}) \mathrm{C}) \mathrm{CC}(=\mathrm{O})[\mathrm{O}-]\end{array}$ & $\begin{array}{l}\text { Part of } \\
\text { options }\end{array}$ & $\begin{array}{l}\text { Part of the } \\
\text { options ( } 2 \\
\text { out of } 2) \text { in } \\
\text { ChemSpider }\end{array}$ \\
\hline Nicotinamide & $\mathrm{C} 6 \mathrm{H} 6 \mathrm{~N} 2 \mathrm{O}$ & 122.048012 & 123.055 & Serum & 8 & $\mathrm{OC}(=\mathrm{N}) \mathrm{c} 1 \mathrm{ccc}$ c 1 & $\begin{array}{l}\text { Matched } \\
\text { (single } \\
\text { option) }\end{array}$ & $\begin{array}{c}\text { Correct } \\
\text { (based on } \\
\text { Pubchem= } \\
\text {-ChemSpider } \\
\text { has an } \\
\text { incorrect } \\
\text { structure) }\end{array}$ \\
\hline Glutamine & C5H10N2O3 & 146.069138 & 147.076 & Serum & 6 & $\mathrm{NC}(=\mathrm{O}) \mathrm{CCC}(\mathrm{C}(=\mathrm{O}) \mathrm{O}) \mathrm{N}$ & $\begin{array}{l}\text { Part of } \\
\text { options }\end{array}$ & $\begin{array}{l}\text { Part of the } \\
\text { options (3 } \\
\text { out of } 15)\end{array}$ \\
\hline $\begin{array}{l}\text { 3-Nitro-L- } \\
\text { Tyrosine }\end{array}$ & C9H10N2O5 & 226.058975 & 277.066 & $\begin{array}{l}\text { STD in } \\
\text { water }\end{array}$ & 13 & $\begin{array}{c}\mathrm{OC}(=\mathrm{O}) \mathrm{C}(\mathrm{Cc} 1 \mathrm{ccc}(\mathrm{c}(\mathrm{c} 1)[\mathrm{N}+] \\
(=\mathrm{O})[\mathrm{O}-]) \mathrm{O}) \mathrm{N}\end{array}$ & $\stackrel{\text { No }}{\text { prediction }}$ & $\begin{array}{l}\text { No } \\
\text { predictions } \\
\text { proposed }\end{array}$ \\
\hline Asparagine & $\mathrm{C} 4 \mathrm{H} 8 \mathrm{~N} 2 \mathrm{O} 3$ & 132.053497 & 133.06 & $\begin{array}{l}\text { STD in } \\
\text { water }\end{array}$ & 5 & $\mathrm{NC}(=\mathrm{O}) \mathrm{CC}(\mathrm{C}(=\mathrm{O}) \mathrm{O}) \mathrm{N}$ & $\begin{array}{l}\text { Part of } \\
\text { options }\end{array}$ & $\begin{array}{l}\text { ureidopropi- } \\
\text { onic acid; no } \\
\text { asparagine }\end{array}$ \\
\hline Glycylglycine & $\mathrm{C} 4 \mathrm{H} 8 \mathrm{~N} 2 \mathrm{O} 3$ & 132.053497 & 133.06 & $\begin{array}{l}\text { STD in } \\
\text { water }\end{array}$ & 3 & $\mathrm{NCC}(=\mathrm{NCC}(=\mathrm{O}) \mathrm{O}) \mathrm{O}$ & $\begin{array}{l}\text { Part of } \\
\text { options }\end{array}$ & $\begin{array}{l}\text { Offers } \\
\text { asparagine, } \\
\text { ureido } \\
\text { propionic } \\
\text { acid and } \\
\text { glycylglycine } \\
\text { between (10) }\end{array}$ \\
\hline Lactose & $\mathrm{C} 12 \mathrm{H} 22 \mathrm{O} 11$ & 342.1162115 & 343.1232 & $\begin{array}{l}\text { STD in } \\
\text { water }\end{array}$ & 9 & $\begin{array}{c}\text { OCC1OC(O)C(C(C1O } \\
\text { C1OC(CO)C(C(C1O)O)O O)O }\end{array}$ & $41 / 54$ & $\begin{array}{l}\text { Identified it } \\
\text { is a sugar }\end{array}$ \\
\hline Cortisol & C21H30O5 & 362.2093241 & 363.2161 & $\begin{array}{l}\text { STD in } \\
\text { water }\end{array}$ & 14 & $\begin{array}{c}\mathrm{OCC}(=\mathrm{O}) \mathrm{C} 1(\mathrm{O}) \mathrm{CCC} 2 \mathrm{C} 1(\mathrm{C}) \\
\mathrm{CC}(\mathrm{O}) \mathrm{C} 1 \mathrm{C} 2 \mathrm{CCC} 2=\mathrm{CC} \\
(=\mathrm{O}) \mathrm{CCC} 12 \mathrm{C}\end{array}$ & 2 out of 4 & $\begin{array}{l}\text { Most } \\
\text { common is } \\
\text { correct }\end{array}$ \\
\hline Corticosterone & C21H30O4 & 346.2144094 & 347.2216 & $\begin{array}{l}\text { STD in } \\
\text { water }\end{array}$ & 10 & $\begin{array}{l}\mathrm{OCC}(=\mathrm{O}) \mathrm{C} 1 \mathrm{CCC} 2 \mathrm{C} 1(\mathrm{C}) \mathrm{CC}(\mathrm{O}) \\
\mathrm{C} 1 \mathrm{C} 2 \mathrm{CCC} 2=\mathrm{CC}(=\mathrm{O}) \mathrm{CCC} 12 \mathrm{C}\end{array}$ & 3 out of 8 & $\begin{array}{l}\text { Most } \\
\text { common is } \\
\text { correct }\end{array}$ \\
\hline Cortisone & $\mathrm{C} 21 \mathrm{H} 28 \mathrm{O} 5$ & 360.193674 & 361.2011 & $\begin{array}{l}\text { STD in } \\
\text { water }\end{array}$ & 16 & $\begin{array}{c}\mathrm{OCC}(=\mathrm{O}) \mathrm{C} 1(\mathrm{O}) \mathrm{CCC} 2 \mathrm{C} 1(\mathrm{C}) \\
\mathrm{CC}(=\mathrm{O}) \mathrm{C} 1 \mathrm{C} 2 \mathrm{CCC} 2=\mathrm{CC}(=\mathrm{O}) \\
\mathrm{CCC} 12 \mathrm{C}\end{array}$ & 2 out of 3 & $\begin{array}{l}\text { Most } \\
\text { common is } \\
\text { correct }\end{array}$ \\
\hline Protirelina & $\mathrm{C} 16 \mathrm{H} 22 \mathrm{~N} 6 \mathrm{O} 4$ & 362.170254 & 363.1767 & $\begin{array}{l}\text { STD in } \\
\text { water }\end{array}$ & 10 & $\begin{array}{c}\mathrm{O}=\mathrm{C} 1 \mathrm{CCC}(\mathrm{N} 1) \mathrm{C}(=\mathrm{O}) \mathrm{NC}(\mathrm{C} \\
(=\mathrm{O}) \mathrm{N} 1 \mathrm{CCCC} 1 \mathrm{C}(=\mathrm{O}) \mathrm{N}) \\
\mathrm{Cc} 1[\mathrm{nH}] \mathrm{cnc} 1\end{array}$ & $\begin{array}{c}\text { No } \\
\text { prediction }\end{array}$ & \\
\hline $\begin{array}{l}\text { Cortisol-21- } \\
\text { acetate }\end{array}$ & $\mathrm{C} 23 \mathrm{H} 32 \mathrm{O} 6$ & 404.21989 & 405.2262 & $\begin{array}{l}\text { STD in } \\
\text { water }\end{array}$ & 12 & $\begin{array}{c}\mathrm{CC}(=\mathrm{O}) \mathrm{OCC}(=\mathrm{O}) \mathrm{C} 1(\mathrm{O}) \mathrm{CCC} 2 \mathrm{C} \\
1(\mathrm{C}) \mathrm{CC}(\mathrm{O}) \mathrm{C} 1 \mathrm{C} 2 \mathrm{CCC} 2=\mathrm{CC} \\
(=\mathrm{O}) \mathrm{CCC} 12 \mathrm{C}\end{array}$ & $\begin{array}{l}\text { with the } \\
\text { correct } \\
\text { formula }\end{array}$ & \\
\hline Rosmarinate & C18H16O8 & 360.0845175 & 361.092 & $\begin{array}{l}\text { STD in } \\
\text { water }\end{array}$ & 11 & $\begin{array}{c}\mathrm{O}=\mathrm{C}(\mathrm{OC}(\mathrm{C}(=\mathrm{O}) \mathrm{O}) \mathrm{Cc} 1 \\
\operatorname{ccc}(\mathrm{c}(\mathrm{c} 1) \mathrm{O}) \mathrm{O}) \mathrm{C}=\mathrm{Cc} 1 \\
\operatorname{ccc}(\mathrm{c}(\mathrm{c} 1) \mathrm{O}) \mathrm{O}\end{array}$ & & \\
\hline $\begin{array}{l}\text { Deoxycorticos- } \\
\text { terone } \\
\text { acetate }\end{array}$ & $\mathrm{C} 23 \mathrm{H} 32 \mathrm{O} 4$ & 372.23006 & 373.2369 & $\begin{array}{l}\text { STD in } \\
\text { water }\end{array}$ & 15 & $\begin{array}{c}\mathrm{CC}(=\mathrm{O}) \mathrm{OCC}(=\mathrm{O}) \mathrm{C} 1 \mathrm{CCC} 2 \mathrm{C} 1 \\
\text { (C)CCC1C2CCC2=C } \\
\mathrm{C}(=\mathrm{O}) \mathrm{CCC} 12 \mathrm{C}\end{array}$ & & \\
\hline Cytosine & $\mathrm{C} 4 \mathrm{H} 5 \mathrm{~N} 3 \mathrm{O}$ & 111.04326 & 112.0505 & Serum & 5 & $\operatorname{Nc1ccnc}(=\mathrm{O})[\mathrm{nH}] 1$ & $\begin{array}{l}\text { Part of } \\
\text { options }\end{array}$ & $\begin{array}{l}1 \text { out of } 17 \\
\text { options } \\
\text { Non-blind } \\
\text { test }\end{array}$ \\
\hline
\end{tabular}




\section{Predicted structures from experimental peak lists of true target cortisol}

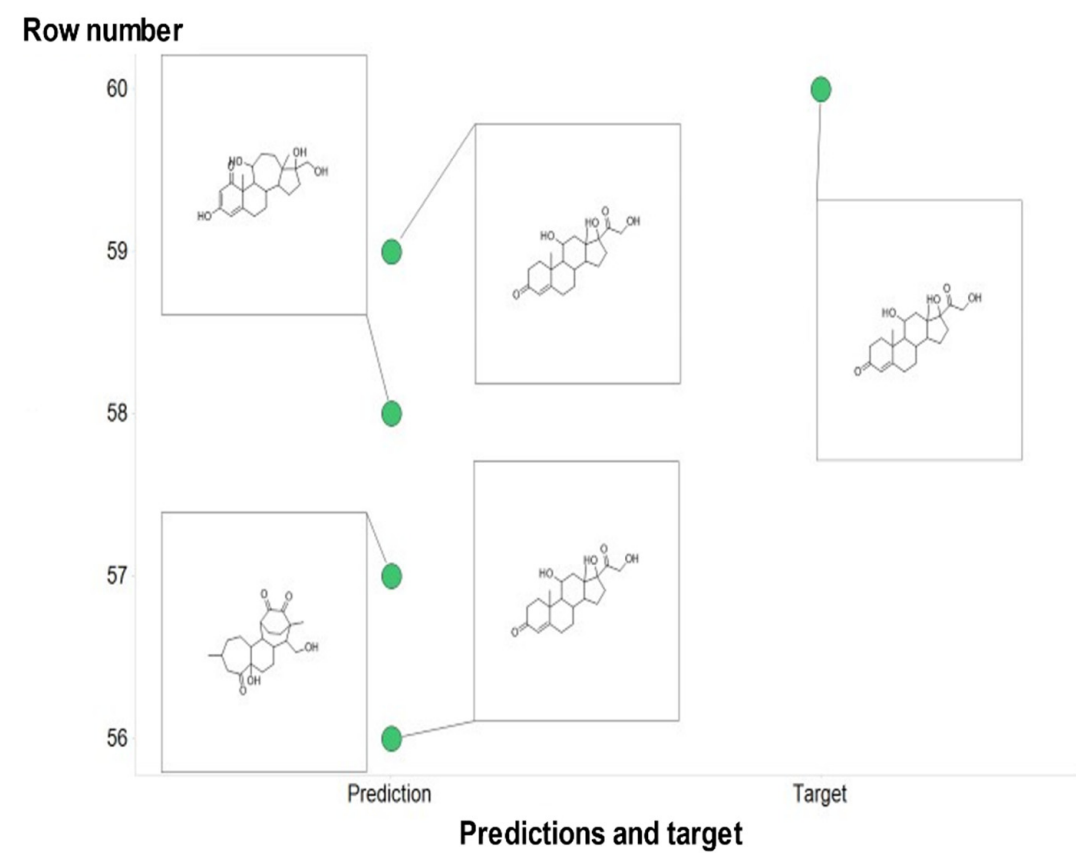

Figure 6. Illustration of the predictive power of MassGenie when presented with an experimental peak list (363.21631, 121.06477, 105.06985, 97.0648, 91.05423, 327.19522, 119.08553, 309.18469, $109.06478,145.10121,93.06989,131.08549,123.08039,79.0542,143.08563)$ of the true molecule cortisol, along with its canonicalized best proposals. In this case $2 / 4$ are correct (and to a chemist's eye far more biologically plausible).

Another means of assessing the ability of systems and algorithms of the present type to predict molecules from mass spectral peak lists is via the so-called CASMI competitions $[30,35,96]$. The last one was in 2017 http://casmi-contest.org/2017/index.shtml (accessed on 1 November 2021), and for our purposes provides, in category 2, 243 natural products (whose identity has now been made available since the end of the competition) and their attendant mass spectral peak lists. We focused on the 93 for which (positive ionisation, MW < 500 Da, SMILES length < 100 characters) on which our model had been trained. On assessing our results (Supplementary Table S1), we found that MassGenie's prediction set had a precisely correct solution in its prediction list in 49 out of 93 challenges $(53 \%)$. In these cases, all the SMILES in the prediction for a particular challenge are isomers of each other. RDKit, by default, first converts any SMILES into a canonical form and then computes the Tanimoto similarity, so there is in effect no ranking. In 14 of the remaining 47 challenges, MassGenie produced a prediction list with the correct chemical formula but, the SMILES string was not right. Due to this, the highest Tanimoto similarity on these challenges was well below 0.8 (which we use as a cutoff for a close similarity [86]) so is taken to be 'unknown'. For the remaining 30 challenges, the MassGenie model did not predict anything that matched the correct chemical formula and we did not pursue these. We note that the best results of the competition itself for challenge 2 http: / / casmi-contest.org/2017/results.shtml (accessed on 1 November 2021) were seemingly $77 / 243$ correct (32\%). On this somewhat post hoc basis, MassGenie would have 'won' the 2017 competition for those molecules for which it had been trained.

Overall, MassGenie performs very creditably, and where it does not one can begin to understand why. First, the experimental spectra often contain spurious peaks due to (unknown) impurities, whereas those generated by FragGenie do not. Secondly, the number of experimental peaks is often really too low to allow a realistic chance of predicting a molecule much beyond those in the training set. Thirdly, there are obvious cases (e.g., long acyl chains) where FragGenie will produce a run of molecules differing by 14(.01565) mass 
units due to the serial loss of -CH2- groups, but the real electrospray mass spectra simply do not mirror this. This said, the fact that MassGenie, augmented where appropriate with VAE-Sim, can produce candidate molecules at all, including those that are not in any library, illustrates the massive power of generative methods of this type in attacking and potentially solving the enormous 'mass-spectrum-to-structure' problem of metabolomics and small molecule analytics.

\section{Discussion}

Artificial neural networks have long been applied in metabolomics [97]. In particular, a great power of modern methods of deep learning is that they do not need to be given explicit rules (though they or their human controllers may certainly benefit from them). The crucial point here, though, is that they are generative, i.e., in our case that they 'automatically' generate molecules in the form of SMILES strings; simple filters can allow the removal of invalid ones or those outside the appropriate mass range. VAE-Sim can be used to add to the list. Although future strategies will be able to incorporate the extensive existing knowledge of mass spectrometrists (e.g., [21,22,98]), the remarkable ability of our system to generate reasonably (and in a great many cases exactly) accurate molecules from candidate mass spectra, without any explicit knowledge of rules for the fragmentation of existing molecules and fragments, must be seen as striking. Note too that we are not directly using $\mathrm{MS}^{\mathrm{n}}$ information based on parent ions beyond the protonated molecular ion at all. In contrast to methods designed to predict the presence of substructures alone, our system seeks to generate the entire molecule of interest, and can do so.

A $10 \times 10$ array of binary (black/white) pixels can take $2^{100}\left(\sim 10^{30}\right)$ forms [10], but few of these represent recognisable or meaningful images. The existence of recognisable 'features' that may be extracted and combined to create meaningful images is what lies at the heart of the kinds of deep learning systems applied in modern, generative image processing [99]. The same is true for natural language processing [78,100]; any 'words' can be generated as strings made from the letters of an alphabet, but relatively few will be 'proper' (i.e., semantically or syntactically meaningful words. In a similar vein, although the number of possible molecules is large, the number of meaningful (and chemically admissible) fragments or substructures from which they are built is far smaller [101]. Equivalently, an infinity of sentences may be constructed from a far smaller number of words, but only a subset of such sentences are meaningful and syntactically or semantically sound $[102,103]$. To this end, we do effectively cast the 'mass-spectrum-to-structure' problem as being equivalent to the kind of sequence-to-sequence language translation problem at which transformers excel, and we here apply them to this problem for the first time. Of the modern, generative methods of deep learning, transformers seem to be especially suited to these kinds of 'language translation' problems, and (for what we believe is the first time in this domain) this is what we have implemented here.

Modern methods in machine learning also recognise that it is possible to train deep networks to extract such features even from unlabelled image or textual inputs. In essence, our system relies similarly upon the use of generative methods with which we can embed molecules in a latent space from which they can be (re)generated. We can regress such latent spaces on any properties of interest. Commonly these latent spaces are used to predict the properties, but here we go in the other direction, where we use in silicogenerated mass spectra to 'predict' the molecules from which they came. The key point is that this is now possible because we can generate them by the million (taking several hours per million on a V100- (not A100-) containing computer, depending on the depth of fragmentation requested). Implicitly, what these deep learning methods do is a form of statistical pattern recognition that effectively learns the rules of language and what letters are likely to be associated with what other letters and in what way. In our case, the ability to generate molecules from mass spectral peaks, i.e., fragments or substructures, shows that the transformer implicitly learns the rules of valency and which fragments may properly be 'bolted together' and how. This approach is in strong contrast (and in the opposite 
direction) to the generation of experimental mass spectra that (i) require access to known standards, (ii) are highly instrument-dependent, (iii) are time-consuming and expensive to acquire, and (iv) are consequently in comparatively short supply.

In the present work, we acquired a large number of molecules encoded as their SMILES strings, and used a gentler (and tuneable) modification of MetFrag [64,65] that we call FragGenie to provide candidate peak lists and paired molecules whence they came. Although we anticipate the strategy to be generic, since this was a proof of concept we confined ourselves to ES+ spectra, and molecules with MW less than 500 and with SMILES encodings of fewer than 100 characters' length. Its ability to predict unseen molecules from FragGenie lists was exceptional, with more than $99 \%$ being seen (after the single-blind code was broken) as having a Tanimoto similarity (using a suitable fingerprint encoding) of more than 0.9 .

Of course, the real 'proof of the pudding' is how our predictions perform when experimental mass spectra are the inputs. In this case, some of the mass spectra contained noise peaks that the FragGenie spectra did not, and these meant that some molecules could not be learned; clearly the test data must bear a reasonable similarity to the training data if the system is to be able to generalise effectively.

Our assessment of the peak lists provided as part of the CASMI 2017 challenge (where the molecular identities are now published) led to an interesting observation, which is that our algorithm either obtained correct results or was unable to make useful suggestions. Algorithms showing an absence of false negatives are known as 'Las Vegas' algorithm [104-106], and our set-up would appear to have that property. Arguably this is very helpful, in that it would be galling in such a hard problem to have a false negative so the 'Las Vegas' property is actually of value in this domain.

Recently, Skinnider et al. [58] applied a recurrent neural network-based generative model to attack a similar problem to that studied here, although they used a billion candidate structures and confined themselves to a test domain of some 2000 psychoactive substances. Although the approaches are not directly comparable, relatively few of their initial structures were accurate, although assessing them against the 'structural priors' in the billion molecules raised this to some $50 \%$. Importantly, they recognised the value of constraining their candidate structures to those that generated the correct molecular ion, and they also showed the value of generative models for this problem.

Although we think that our strategy represents an excellent start, we should point out that this success did require what for an academic biology laboratory is a substantial computing resource-the DGX A100 8-GPU system we used allowed us to train a 400-million node network, which would in fact have represented a world record for the largest published deep learning network less than three years ago [79]. (This said, the current record, writing originally in June 2021, is more than three and almost four orders of magnitude greater $[75,79,107]$.) It is clear that the effectiveness of such networks will continue to increase, since how this potency scales with the size of the training dataset and of the network is broadly predictable [107-111]). We also recognise that MassGenie can be improved much further. First, although we have trained our system on millions of molecules, what is learned does tend to scale with the amount of material on which it is trained [112]. Indeed, there are already $~ 11$ billion molecules in the ZINC database, and (as has already been done by Reymond and colleagues [45]) we can generate as many more (in both type and number) as we like in silico; using these in further training, probably with larger encoding networks, will require more computer power, but this is evidently going to become available (as noted above, e.g., in the training of GPT-3 [75] and others [113]). Secondly, the rules we have used in the in silico fragmentation are extremely crude and rudimentary; they can certainly bear refining or replacing with some of the more knowledge-based in silico fragmentation methods available in commercial software (which would have to have an API to admit the generation of large numbers of SMILES-spectrum pairs, as here). Thirdly, we have not used any knowledge of isotope patterns and the like, relying solely on exact masses to discriminate the likely atoms in a fragment. Fourthly, we have used SMILES 
strings as our molecular encoding; a move to graphical representations [114-119] is likely to prove beneficial. Fifthly, we have yet to apply equivalent strategies to negative ionisation spectra and to larger molecules, and to a far greater number of molecules containing $\mathrm{P}$ and S. Sixthly, by using canonical SMILES we ignore-and thus cannot discriminate-any stereoisomerism. Finally, we did not really explore at all the many flavours of deep network being developed to learn, store and transform information [120,121]. Although this is a very fast-moving field, other and more efficient varieties of generative methods based on transformers (e.g., [77,122-133]), as we use in the spectrum-to-structure problem for the first time here, do presently seem particularly promising. In line with the title of the original transformer paper [77], we might conclude by commenting that "accurate fragmentation is all you need".

Supplementary Materials: The following are available online at https:/ /www.mdpi.com/article/ 10.3390/biom11121793/s1, Table S1: File test_set_data_analysis.xlsx representing the peak lists and molecules shown in Figure 3.

Author Contributions: A.D.S. wrote the transformer code, N.S. wrote FragGenie, S.S. wrote and applied VAE-Sim. N.S. and S.S. constructed the datasets. I.R. and M.W.M. ran experimental mass spectra and provided peak lists and cheminformatic analyses. D.B.K. conceived the project, obtained funding for it, and wrote the first draft of the manuscript. All authors contributed to further drafting and editing, and approved the final version. All authors have read and agreed to the published version of the manuscript.

Funding: ADS was funded via the University of Liverpool. SS and DBK are funded as part of the UK EPSRC project SuSCoRD (EP/S004963/1), partly sponsored by Akzo-Nobel. DBK is also funded by the Novo Nordisk Foundation (grant NNF20CC0035580). The DGX-A100 computer was purchased as part of a UK BBSRC infrastructure grant to the University of Liverpool GeneMill. The APC was waived by the journal.

Institutional Review Board Statement: Not applicable.

Informed Consent Statement: Not applicable.

Data Availability Statement: Not applicable.

Acknowledgments: We thank Pete Balshaw (Scan Computers) and Cliff Addison (University of Liverpool) for their help in the specification and installation of the DGX-A100 computer.

Conflicts of Interest: The authors declare that they have no conflict of interest.

\section{References}

1. Griffin, J.L. The Cinderella story of metabolic profiling: Does metabolomics get to go to the functional genomics ball? Philos. Trans. R. Soc. Lond. B Biol. Sci. 2006, 361, 147-161. [CrossRef] [PubMed]

2. Oliver, S.G.; Winson, M.K.; Kell, D.B.; Baganz, F. Systematic functional analysis of the yeast genome. Trends Biotechnol. 1998, 16, 373-378. [CrossRef]

3. Dunn, W.B.; Broadhurst, D.; Begley, P.; Zelena, E.; Francis-McIntyre, S.; Anderson, N.; Brown, N.; Knowles, J.; Halsall, A.; Haselden, J.N.; et al. The Husermet consortium, Procedures for large-scale metabolic profiling of serum and plasma using gas chromatography and liquid chromatography coupled to mass spectrometry. Nat. Protoc. 2011, 6, 1060-1083. [CrossRef]

4. Dunn, W.B.; Erban, A.; Weber, R.J.M.; Creek, D.J.; Brown, M.; Breitling, R.; Hankemeier, T.; Goodacre, R.; Neumann, S.; Kopka, J.; et al. Mass Appeal: Metabolite identification in mass spectrometry-focused untargeted metabolomics. Metabolites 2013, 9, S44-S66. [CrossRef]

5. Arús-Pous, J.; Awale, M.; Probst, D.; Reymond, J.L. Exploring Chemical Space with Machine Learning. Chimia 2019, 73, 1018-1023. [CrossRef]

6. Bohacek, R.S.; McMartin, C.; Guida, W.C. The art and practice of structure-based drug design: A molecular modeling perspective. Med. Res. Rev. 1996, 16, 3-50. [CrossRef]

7. Polishchuk, P.G.; Madzhidov, T.I.; Varnek, A. Estimation of the size of drug-like chemical space based on GDB-17 data. J. Comput. Aided Mol. Des. 2013, 27, 675-679. [CrossRef]

8. Sterling, T.; Irwin, J.J. ZINC 15-Ligand Discovery for Everyone. J. Chem. Inf. Model. 2015, 55, 2324-2337. [CrossRef]

9. Pitt, W.R.; Parry, D.M.; Perry, B.G.; Groom, C.R. Heteroaromatic Rings of the Future. J. Med. Chem. 2009, 52, $2952-2963$. [CrossRef] [PubMed] 
10. Hastie, T.; Tibshirani, R.; Friedman, J. The Elements of Statistical Learning: Data Mining, Inference and Prediction, 2nd ed.; Springer: Berlin, Germany, 2009.

11. Nash, W.J.; Dunn, W.B. From mass to metabolite in human untargeted metabolomics: Recent advances in annotation of metabolites applying liquid chromatography-mass spectrometry data. Trends Anal. Chem. 2019, 120, 115324. [CrossRef]

12. Sindelar, M.; Patti, G.J. Chemical Discovery in the Era of Metabolomics. J. Am. Chem. Soc. 2020, 142, 9097-9105. [CrossRef] [PubMed]

13. Shen, X.; Wang, R.; Xiong, X.; Yin, Y.; Cai, Y.; Ma, Z.; Liu, N.; Zhu, Z.J. Metabolic reaction network-based recursive metabolite annotation for untargeted metabolomics. Nat. Commun. 2019, 10, 1516. [CrossRef]

14. Misra, B.B.; van der Hooft, J.J.J. Updates in metabolomics tools and resources: 2014-2015. Electrophoresis 2016, 37, 86-110. [CrossRef] [PubMed]

15. Misra, B.B. New software tools, databases, and resources in metabolomics: Updates from 2020. J. Metab. 2021, 17, 49. [CrossRef]

16. Dunn, W.B.; Lin, W.; Broadhurst, D.; Begley, P.; Brown, M.; Zelena, E.; Vaughan, A.A.; Halsall, A.; Harding, N.; Knowles, J.D.; et al. Molecular phenotyping of a UK population: Defining the human serum metabolome. J. Metab. 2015, 11, 9-26. [CrossRef] [PubMed]

17. Ganna, A.; Fall, T.; Salihovic, S.; Lee, W.; Broeckling, C.D.; Kumar, J.; Hägg, S.; Stenemo, M.; Magnusson, P.K.E.; Prenni, J.E.; et al. Large-scale non-targeted metabolomic profiling in three human population-based studies. J. Metab. 2016, 12, 4. [CrossRef]

18. Wright Muelas, M.; Roberts, I.; Mughal, F.; O’Hagan, S.; Day, P.J.; Kell, D.B. An untargeted metabolomics strategy to measure differences in metabolite uptake and excretion by mammalian cell lines. J. Metab. 2020, 16, 107. [CrossRef]

19. Borges, R.M.; Colby, S.M.; Das, S.; Edison, A.S.; Fiehn, O.; Kind, T.; Lee, J.; Merrill, A.T.; Merz, K.M., Jr.; Metz, T.O.; et al. Quantum Chemistry Calculations for Metabolomics. Chem. Rev. 2021, 121, 5633-5670. [CrossRef]

20. Peisl, B.Y.L.; Schymanski, E.L.; Wilmes, P. Dark matter in host-microbiome metabolomics: Tackling the unknowns-A review. Anal. Chim. Acta 2018, 1037, 13-27. [CrossRef] [PubMed]

21. De Vijlder, T.; Valkenborg, D.; Lemiere, F.; Romijn, E.P.; Laukens, K.; Cuyckens, F. A tutorial in small molecule identification via electrospray ionization-mass spectrometry: The practical art of structural elucidation. Mass Spectrom. Rev. 2018, 37, 607-629. [CrossRef]

22. Dührkop, K.; Fleischauer, M.; Ludwig, M.; Aksenov, A.A.; Melnik, A.V.; Meusel, M.; Dorrestein, P.C.; Rousu, J.; Böcker, S. SIRIUS 4: A rapid tool for turning tandem mass spectra into metabolite structure information. Nat. Methods 2019, 16, 299-302. [CrossRef]

23. Kind, T.; Tsugawa, H.; Cajka, T.; Ma, Y.; Lai, Z.; Mehta, S.S.; Wohlgemuth, G.; Barupal, D.K.; Showalter, M.R.; Arita, M.; et al. Identification of small molecules using accurate mass MS/MS search. Mass Spectrom. Rev. 2018, 37, 513-532. [CrossRef]

24. Vinaixa, M.; Schymanski, E.L.; Neumann, S.; Navarro, M.; Salek, R.M.; Yanes, O. Mass spectral databases for LC/MS- and GC/MS-based metabolomics: State of the field and future prospects. Trends Anal. Chem. 2016, 78, 23-35. [CrossRef]

25. Neumann, S.; Bocker, S. Computational mass spectrometry for metabolomics: Identification of metabolites and small molecules. Anal. Bioanal. Chem. 2010, 398, 2779-2788. [CrossRef]

26. Blaženović, I.; Kind, T.; Ji, J.; Fiehn, O. Software Tools and Approaches for Compound Identification of LC-MS/MS Data in Metabolomics. Metabolites 2018, 8, 31. [CrossRef]

27. Creek, D.J.; Dunn, W.B.; Fiehn, O.; Griffin, J.L.; Hall, R.D.; Lei, Z.; Mistrik, R.; Neumann, S.; Schymanski, E.L.; Sumner, L.W.; et al. Metabolite identification: Are you sure? And how do your peers gauge your confidence? Metabolites 2014, 10, 350-353. [CrossRef]

28. Peters, K.; Bradbury, J.; Bergmann, S.; Capuccini, M.; Cascante, M.; de Atauri, P.; Ebbels, T.M.D.; Foguet, C.; Glen, R.; GonzalezBeltran, A.; et al. PhenoMeNal: Processing and analysis of metabolomics data in the cloud. Gigascience 2019, 8, giy149. [CrossRef] [PubMed]

29. Bingol, K.; Bruschweiler-Li, L.; Li, D.; Zhang, B.; Xie, M.; Brüschweiler, R. Emerging new strategies for successful metabolite identification in metabolomics. Bioanalysis 2016, 8, 557-573. [CrossRef]

30. Blaženović, I.; Kind, T.; Torbašinović, H.; Obrenović, S.; Mehta, S.S.; Tsugawa, H.; Wermuth, T.; Schauer, N.; Jahn, M.; Biedendieck, R.; et al. Comprehensive comparison of in silico MS/MS fragmentation tools of the CASMI contest: Database boosting is needed to achieve 93\% accuracy. J. Cheminform. 2017, 9, 32. [CrossRef] [PubMed]

31. Djoumbou-Feunang, Y.; Pon, A.; Karu, N.; Zheng, J.; Li, C.; Arndt, D.; Gautam, M.; Allen, F.; Wishart, D.S. CFM-ID 3.0: Significantly Improved ESI-MS/MS Prediction and Compound Identification. Metabolites 2019, 9, 72. [CrossRef] [PubMed]

32. Djoumbou-Feunang, Y.; Fiamoncini, J.; Gil-de-la-Fuente, A.; Greiner, R.; Manach, C.; Wishart, D.S. BioTransformer: A comprehensive computational tool for small molecule metabolism prediction and metabolite identification. J. Cheminform. 2019, 11, 2. [CrossRef]

33. Alexandrov, T. Spatial Metabolomics and Imaging Mass Spectrometry in the Age of Artificial Intelligence. Annu. Rev. Biomed. Data Sci. 2020, 3, 61-87. [CrossRef] [PubMed]

34. Ludwig, M.; Nothias, L.-F.; Dührkop, K.; Koester, I.; Fleischauer, M.; Hoffmann, M.A.; Petras, D.; Vargas, F.; Morsy, M.; Aluwihare, L.; et al. Database-independent molecular formula annotation using Gibbs sampling through ZODIAC. Nat. Mach. Intell. 2020, 2, 629-641. [CrossRef]

35. McEachran, A.D.; Chao, A.; Al-Ghoul, H.; Lowe, C.; Grulke, C.; Sobus, J.R.; Williams, A.J. Revisiting Five Years of CASMI Contests with EPA Identification Tools. Metabolites 2020, 10, 260. [CrossRef]

36. Bowen, B.P.; Northen, T.R. Dealing with the unknown: Metabolomics and metabolite atlases. J. Am. Soc. Mass Spectrom. 2010, 21, 1471-1476. [CrossRef] 
37. Bhatia, A.; Sarma, S.J.; Lei, Z.; Sumner, L.W. UHPLC-QTOF-MS/MS-SPE-NMR: A Solution to the Metabolomics Grand Challenge of Higher-Throughput, Confident Metabolite Identifications. Methods Mol. Biol. 2019, 2037, 113-133. [PubMed]

38. Liu, Y.; De Vijlder, T.; Bittremieux, W.; Laukens, K.; Heyndrickx, W. Current and future deep learning algorithms for tandem mass spectrometry (MS/MS)-based small molecule structure elucidation. Rapid Commun. Mass Spectrom. 2021, e9120. [CrossRef] [PubMed]

39. Tripathi, A.; Vázquez-Baeza, Y.; Gauglitz, J.M.; Wang, M.; Dührkop, K.; Nothias-Esposito, M.; Acharya, D.D.; Ernst, M.; van der Hooft, J.J.J.; Zhu, Q.; et al. Chemically informed analyses of metabolomics mass spectrometry data with Qemistree. Nat. Chem. Biol. 2021, 17, 146-151. [CrossRef]

40. Stravs, M.A.; Dührkop, K.; Böcker, S.; Zamboni, N. MSNovelist: De novo structure generation from mass spectra. bioRxiv 2021, 450875.

41. Buchanan, B.G.; Feigenbaum, E.A. DENDRAL and META-DENDRAL: Their application dimensions. Artif. Intell. 1978, 11, 5-24. [CrossRef]

42. Feigenbaum, E.A.; Buchanan, B.G. DENDRAL and META-DENDRAL: Roots of knowledge systems and expert system applications. Artif. Intell. 1993, 59, 223-240. [CrossRef]

43. Lindsay, R.K.; Buchanan, B.G.; Feigenbaum, E.A.; Lederberg, J. DENDRAL—A Case study of the first expert system for scientific hypothesis formation. Artif. Intell. 1993, 61, 209-261. [CrossRef]

44. Kell, D.B.; Oliver, S.G. Here is the evidence, now what is the hypothesis? The complementary roles of inductive and hypothesisdriven science in the post-genomic era. Bioessays 2004, 26, 99-105. [CrossRef] [PubMed]

45. Ruddigkeit, L.; van Deursen, R.; Blum, L.C.; Reymond, J.L. Enumeration of 166 billion organic small molecules in the chemical universe database GDB-17. J. Chem. Inf. Model. 2012, 52, 2864-2875. [CrossRef]

46. Gómez-Bombarelli, R.; Wei, J.N.; Duvenaud, D.; Hernández-Lobato, J.M.; Sánchez-Lengeling, B.; Sheberla, D.; AguileraIparraguirre, J.; Hirzel, T.D.; Adams, R.P.; Aspuru-Guzik, A. Automatic Chemical Design Using a Data-Driven Continuous Representation of Molecules. ACS Cent. Sci. 2018, 4, 268-276. [CrossRef]

47. LeCun, Y.; Bengio, Y.; Hinton, G. Deep learning. Nature 2015, 521, 436-444. [CrossRef]

48. Schmidhuber, J. Deep learning in neural networks: An overview. Neural Netw. 2015, 61, 85-117. [CrossRef]

49. Arús-Pous, J.; Blaschke, T.; Ulander, S.; Reymond, J.L.; Chen, H.; Engkvist, O. Exploring the GDB-13 chemical space using deep generative models. J. Cheminform. 2019, 11, 20. [CrossRef]

50. David, L.; Arús-Pous, J.; Karlsson, J.; Engkvist, O.; Bjerrum, E.J.; Kogej, T.; Kriegl, J.M.; Beck, B.; Chen, H. Applications of Deep-Learning in Exploiting Large-Scale and Heterogeneous Compound Data in Industrial Pharmaceutical Research. Front. Pharm. 2019, 10, 1303. [CrossRef]

51. Grisoni, F.; Schneider, G. De novo Molecular Design with Generative Long Short-term Memory. Chimia 2019, 73 , $1006-1011$. [CrossRef]

52. Schneider, G. Generative models for artificially-intelligent molecular design. Mol. Inform. 2018, 37, 188031. [CrossRef] [PubMed]

53. Sanchez-Lengeling, B.; Aspuru-Guzik, A. Inverse molecular design using machine learning: Generative models for matter engineering. Science 2018, 361, 360-365. [CrossRef]

54. Segler, M.H.S.; Kogej, T.; Tyrchan, C.; Waller, M.P. Generating Focused Molecule Libraries for Drug Discovery with Recurrent Neural Networks. ACS Cent. Sci. 2018, 4, 120-131. [CrossRef] [PubMed]

55. Elton, D.C.; Boukouvalas, Z.; Fuge, M.D.; Chung, P.W. Deep learning for molecular design: A review of the state of the art. Mol. Syst. Des. Eng. 2019, 4, 828-849. [CrossRef]

56. Kell, D.B.; Samanta, S.; Swainston, N. Deep learning and generative methods in cheminformatics and chemical biology: Navigating small molecule space intelligently. J. Biochem. 2020, 477, 4559-4580. [CrossRef]

57. Jiménez-Luna, J.; Grisoni, F.; Weskamp, N.; Schneider, G. Artificial intelligence in drug discovery: Recent advances and future perspectives. Expert Opin. Drug Discov. 2021, 16, 949-959. [CrossRef]

58. Skinnider, M.; Wang, F.; Pasin, D.; Greiner, R.; Foster, L.; Dalsgaard, P.; Wishart, D.S. A Deep Generative Model Enables Automated Structure Elucidation of Novel Psychoactive Substances. ChemRxiv 2021, 1-23. [CrossRef]

59. Bepler, T.; Berger, B. Learning the protein language: Evolution, structure, and function. Cell Syst. 2021, 12, 654-666.e3. [CrossRef] [PubMed]

60. Samanta, S.; O’Hagan, S.; Swainston, N.; Roberts, T.J.; Kell, D.B. VAE-Sim: A novel molecular similarity measure based on a variational autoencoder. Molecules 2020, 25, 3446. [CrossRef]

61. Grimme, S. Towards first principles calculation of electron impact mass spectra of molecules. Angew. Chem. Int. Ed. Engl. 2013, 52, 6306-6312. [CrossRef]

62. Scheubert, K.; Hufsky, F.; Böcker, S. Computational mass spectrometry for small molecules. J. Cheminform. 2013, 5, 12. [CrossRef]

63. Ridder, L.; van der Hooft, J.J.J.; Verhoeven, S. Automatic Compound Annotation from Mass Spectrometry Data Using MAGMa. J. Mass Spectrom. 2014, 3, S0033. [CrossRef]

64. Ruttkies, C.; Schymanski, E.L.; Wolf, S.; Hollender, J.; Neumann, S. MetFrag relaunched: Incorporating strategies beyond in silico fragmentation. J. Cheminform 2016, 8, 3. [CrossRef]

65. Ruttkies, C.; Neumann, S.; Posch, S. Improving MetFrag with statistical learning of fragment annotations. BMC Bioinform. 2019, 20, 376. [CrossRef] 
66. da Silva, R.R.; Wang, M.; Nothias, L.F.; van der Hooft, J.J.J.; Caraballo-Rodríguez, A.M.; Fox, E.; Balunas, M.J.; Klassen, J.L.; Lopes, N.P.; Dorrestein, P.C. Propagating annotations of molecular networks using in silico fragmentation. PLoS Comput. Biol. 2018, 14, e1006089. [CrossRef] [PubMed]

67. Wandy, J.; Davies, V.; van der Hooft, J.J.J.; Weidt, S.; Daly, R.; Rogers, S. In Silico Optimization of Mass Spectrometry Fragmentation Strategies in Metabolomics. Metabolites 2019, 9, 219. [CrossRef] [PubMed]

68. Ernst, M.; Kang, K.B.; Caraballo-Rodriguez, A.M.; Nothias, L.F.; Wandy, J.; Chen, C.; Wang, M.; Rogers, S.; Medema, M.H.; Dorrestein, P.C.; et al. MolNetEnhancer: Enhanced Molecular Networks by Integrating Metabolome Mining and Annotation Tools. Metabolites 2019, 9, 144. [CrossRef] [PubMed]

69. Allen, F.; Pon, A.; Wilson, M.; Greiner, R.; Wishart, D. CFM-ID: A web server for annotation, spectrum prediction and metabolite identification from tandem mass spectra. Nucleic Acids Res. 2014, 42, W94-W99. [CrossRef]

70. Schüler, J.A.; Neumann, S.; Müller-Hannemann, M.; Brandt, W. ChemFrag: Chemically meaningful annotation of fragment ion mass spectra. J. Mass Spectrom. 2018, 53, 1104-1115. [CrossRef]

71. Hoffmann, M.A.; Nothias, L.F.; Ludwig, M.; Fleischauer, M.; Gentry, E.C.; Witting, M.; Dorrestein, P.C.; Dührkop, K.; Böcker, S. High-confidence structural annotation of metabolites absent from spectral libraries. Nat. Biotechnol. 2021. [CrossRef]

72. Feunang, Y.D.; Eisner, R.; Knox, C.; Chepelev, L.; Hastings, J.; Owen, G.; Fahy, E.; Steinbeck, C.; Subramanian, S.; Bolton, E.; et al. ClassyFire: Automated chemical classification with a comprehensive, computable taxonomy. J. Cheminform. $2016,8,61$.

73. Hassanpour, N.; Alden, N.; Menon, R.; Jayaraman, A.; Lee, K.; Hassoun, S. Biological Filtering and Substrate Promiscuity Prediction for Annotating Untargeted Metabolomics. Metabolites 2020, 10, 160. [CrossRef] [PubMed]

74. Krizhevsky, A.; Sutskever, I.; Hinton, G.E. ImageNet Classification with Deep Convolutional Neural Networks. Adv. Neural Inf. Process. Syst. 2012, 25, 1090-1098. [CrossRef]

75. Brown, T.B.; Mann, B.; Ryder, N.; Subbiah, M.; Kaplan, J.; Dhariwal, P.; Neelakantan, A.; Shyam, P.; Sastry, G.; Askell, A.; et al. Language Models are Few-Shot Learners. arXiv 2020, arXiv:2005.14165.

76. Shardlow, M.; Ju, M.; Li, M.; O’Reilly, C.; Iavarone, E.; McNaught, J.; Ananiadou, S. A Text Mining Pipeline Using Active and Deep Learning Aimed at Curating Information in Computational Neuroscience. Neuroinformatics 2019, 17, 391-406. [CrossRef] [PubMed]

77. Vaswani, A.; Shazeer, N.; Parmar, N.; Uszkoreit, J.; Jones, L.; Gomez, A.N.; Kaiser, L.; Polosukhin, I. Attention Is All You Need. arXiv 2017, arXiv:1706.03762.

78. Devlin, J.; Chang, M.-W.; Lee, K.; Toutanova, K. BERT: Pre-training of Deep Bidirectional Transformers for Language Understanding. arXiv 2018, arXiv:1810.04805.

79. Hutson, M. The language machines. Nature 2021, 591, 22-25. [CrossRef]

80. Singh, S.; Mahmood, A. The NLP Cookbook: Modern Recipes for Transformer based Deep Learning Architectures. arXiv 2021, arXiv:2104.10640. [CrossRef]

81. Topal, M.O.; Bas, A.; van Heerden, I. Exploring Transformers in Natural Language Generation: GPT, BERT, and XLNet. arXiv 2021, arXiv:2102.08036.

82. Kaiser, L.; Gomez, A.N.; Shazeer, N.; Vaswani, A.; Parmar, N.; Jones, L.; Uszkoreit, J. One Model To Learn Them All. arXiv 2017, arXiv:1706.05137.

83. Lu, K.; Grover, A.; Abbeel, P.; Mordatch, I. Pretrained Transformers as Universal Computation Engines. arXiv 2021, arXiv:2103.05247.

84. Shrivastava, A.D.; Swainston, N.; Samanta, S.; Roberts, I.; Wright Muelas, M.; Kell, D.B. MassGenie: A transformer-based deep learning method for identifying small molecules from their mass spectra. bioRxiv 2021. [CrossRef]

85. O'Hagan, S.; Swainston, N.; Handl, J.; Kell, D.B. A 'rule of $0.5^{\prime}$ for the metabolite-likeness of approved pharmaceutical drugs. Metabolites 2015, 11, 323-339. [CrossRef]

86. O'Hagan, S.; Kell, D.B. Consensus rank orderings of molecular fingerprints illustrate the 'most genuine' similarities between marketed drugs and small endogenous human metabolites, but highlight exogenous natural products as the most important 'natural' drug transporter substrates. ADMET DMPK 2017, 5, 85-125.

87. Roberts, I.; Wright Muelas, M.; Taylor, J.M.; Davison, A.S.; Xu, Y.; Grixti, J.M.; Gotts, N.; Sorokin, A.; Goodacre, R.; Kell, D.B. Untargeted metabolomics of COVID-19 patient serum reveals potential prognostic markers of both severity and outcome. medRxiv 2020. [CrossRef]

88. Willighagen, E.L.; Mayfield, J.W.; Alvarsson, J.; Berg, A.; Carlsson, L.; Jeliazkova, N.; Kuhn, S.; Pluskal, T.; Rojas-Cherto, M.; Spjuth, O.; et al. The Chemistry Development Kit (CDK) v2.0: Atom typing, depiction, molecular formulas, and substructure searching. J. Cheminform. 2017, 9, 33. [CrossRef] [PubMed]

89. Goyal, P.; Dollár, P.; Girshick, R.; Noordhuis, P.; Wesolowski, L.; Kyrola, A.; Tulloch, A.; Jia, Y.; He, K. Accurate, Large Minibatch SGD: Training ImageNet in 1 Hour. arXiv 2017, arXiv:1706.02677.

90. Sumner, L.W.; Amberg, A.; Barrett, D.; Beale, M.H.; Beger, R.; Daykin, C.A.; Fan, T.W.; Fiehn, O.; Goodacre, R.; Griffin, J.L.; et al. Proposed minimum reporting standards for chemical analysis Chemical Analysis Working Group (CAWG) Metabolomics Standards Initiative (MSI). Metabolites 2007, 3, 211-221.

91. Bender, A.; Glen, R.C. Molecular similarity: A key technique in molecular informatics. Org. Biomol. Chem. 2004, 2, 3204-3218. [CrossRef] [PubMed] 
92. Maggiora, G.; Vogt, M.; Stumpfe, D.; Bajorath, J. Molecular Similarity in Medicinal Chemistry. J. Med. Chem. 2014, 57, 3186-3204. [CrossRef] [PubMed]

93. Todeschini, R.; Consonni, V.; Xiang, H.; Holliday, J.; Buscema, M.; Willett, P. Similarity Coefficients for Binary Chemoinformatics Data: Overview and Extended Comparison Using Simulated and Real Data Sets. J. Chem. Inf. Model. 2012, 52, $2884-2901$. [CrossRef] [PubMed]

94. Jeffryes, J.G.; Colastani, R.L.; Elbadawi-Sidhu, M.; Kind, T.; Niehaus, T.D.; Broadbelt, L.J.; Hanson, A.D.; Fiehn, O.; Tyo, K.E.; Henry, C.S. MINEs: Open access databases of computationally predicted enzyme promiscuity products for untargeted metabolomics. J. Cheminform. 2015, 7, 44. [CrossRef] [PubMed]

95. Wu, H.; Zhou, J. Privacy Leakage of SIFT Features via Deep Generative Model based Image Reconstruction. arXiv 2020, arXiv:2009.01030. [CrossRef]

96. Schymanski, E.L.; Neumann, S. The Critical Assessment of Small Molecule Identification (CASMI): Challenges and Solutions. Metabolites 2013, 3, 517-538. [CrossRef] [PubMed]

97. Mendez, K.M.; Broadhurst, D.I.; Reinke, S.N. The application of artificial neural networks in metabolomics: A historical perspective. Metabolites 2019, 15, 142. [CrossRef] [PubMed]

98. Kind, T.; Fiehn, O. Seven Golden Rules for heuristic filtering of molecular formulas obtained by accurate mass spectrometry. BMC Bioinform. 2007, 8, 105. [CrossRef]

99. Singh, A.; Sengupta, S.; Lakshminarayanan, V. Explainable deep learning models in medical image analysis. arXiv 2020, arXiv:2005.13799.

100. Trieu, H.L.; Tran, T.T.; Duong, K.N.A.; Nguyen, A.; Miwa, M.; Ananiadou, S. DeepEventMine: End-to-end neural nested event extraction from biomedical texts. Bioinformatics 2020, 36, 4910-4917. [CrossRef]

101. Ertl, P. Cheminformatics analysis of organic substituents: Identification of the most common substituents, calculation of substituent properties, and automatic identification of drug-like bioisosteric groups. J. Chem Inf. Comput. Sci. 2003, 43, 374-380. [CrossRef]

102. Ananiadou, S.; Kell, D.B.; Tsujii, J.-I. Text Mining and its potential applications in Systems Biology. Trends Biotechnol. 2006, 24, 571-579. [CrossRef]

103. Ju, M.; Nguyen, N.T.H.; Miwa, M.; Ananiadou, S. An ensemble of neural models for nested adverse drug events and medication extraction with subwords. J. Am. Med. Inform. Assoc. 2019, 27, 22-30. [CrossRef] [PubMed]

104. Babai, L. Monte-Carlo Algorithms in Graph Isomorphism Testing; D.M.S. No. 79-10; University De Montréal: Montréal, QC, Canada, 1979.

105. Luby, M.; Sinclair, A.; Zuckerman, D. Optimal speedup of Las Vegas algorithms. Inf. Proc. Lett. 1993, 47, 173-180. [CrossRef]

106. Sze, S.H.; Pevzner, P.A. Las Vegas algorithms for gene recognition: Suboptimal and error-tolerant spliced alignment. J. Comput. Biol. 1997, 4, 297-309. [CrossRef]

107. Zhai, X.; Kolesnikov, A.; Houlsby, N.; Beyer, L. Scaling Vision Transformers. arXiv 2021, arXiv:2106.04560.

108. Sun, C.; Shrivastava, A.; Singh, S.; Gupta, A. Revisiting Unreasonable Effectiveness of Data in Deep Learning Era. arXiv 2017, arXiv:1707.02968.

109. Kaplan, J.; McCandlish, S.; Henighan, T.; Brown, T.B.; Chess, B.; Child, R.; Gray, S.; Radford, A.; Wu, J.; Amodei, D. Scaling Laws for Neural Language Models. arXiv 2020, arXiv:2001.08361.

110. Henighan, T.; Kaplan, J.; Katz, M.; Chen, M.; Hesse, C.; Jackson, J.; Jun, H.; Brown, T.B.; Dhariwal, P.; Gray, S.; et al. Scaling Laws for Autoregressive Generative Modeling. arXiv 2020, arXiv:2010.14701.

111. Sharma, U.; Kaplan, J. A Neural Scaling Law from the Dimension of the Data Manifold. arXiv 2021, arXiv:2004.10802.

112. Domingos, P. Every Model Learned by Gradient Descent Is Approximately a Kernel Machine. arXiv 2020, arXiv:2012.00152.

113. Fedus, W.; Zoph, B.; Shazeer, N. Switch Transformers: Scaling to Trillion Parameter Models with Simple and Efficient Sparsity. arXiv 2021, arXiv:2101.03961.

114. Dwivedi, V.P.; Joshi, C.K.; Laurent, T.; Bengio, Y.; Bresson, X. Benchmarking Graph Neural Networks. arXiv 2020, arXiv:2003.00982.

115. Khemchandani, Y.; O’Hagan, S.; Samanta, S.; Swainston, N.; Roberts, T.J.; Bollegala, D.; Kell, D.B. DeepGraphMolGen, a multiobjective, computational strategy for generating molecules with desirable properties: A graph convolution and reinforcement learning approach. J. Cheminform. 2020, 12, 53. [CrossRef]

116. Lim, J.; Hwang, S.Y.; Moon, S.; Kim, S.; Kim, W.Y. Scaffold-based molecular design with a graph generative model. Chem. Sci. 2020, 11, 1153-1164. [CrossRef] [PubMed]

117. Shi, C.; Xu, M.; Zhu, Z.; Zhang, W.; Zhang, M.; Tang, J. GraphAF: A Flow-based Autoregressive Model for Molecular Graph Generation. arXiv 2020, arXiv:2001.09382.

118. Wu, Z.; Pan, S.; Chen, F.; Long, G.; Zhang, C.; Yu, P.S. A Comprehensive Survey on Graph Neural Networks. IEEE Trans. Neural Netw. Learn. Syst. 2020, 32, 4-24. [CrossRef]

119. David, L.; Thakkar, A.; Mercado, R.; Engkvist, O. Molecular representations in AI-driven drug discovery: A review and practical guide. J. Cheminform. 2020, 12, 56. [CrossRef]

120. Elsken, T.; Metzen, J.H.; Hutter, F. Neural Architecture Search: A Survey. arXiv 2018, arXiv:1808.05377.

121. Pham, H.; Guan, M.Y.; Zoph, B.; Le, Q.V.; Dean, J. Efficient Neural Architecture Search via Parameter Sharing. arXiv 2018, arXiv:1802.03268. 
122. Chithrananda, S.; Grand, G.; Ramsundar, B. ChemBERTa: Large-Scale Self-Supervised Pretraining for Molecular Property Prediction. arXiv 2020, arXiv:2010.09885.

123. Tay, Y.; Dehghani, M.; Bahri, D.; Metzler, D. Efficient Transformers: A Survey. arXiv 2020, arXiv:2009.06732v2.

124. Lin, T.; Wang, Y.; Liu, X.; Qiu, X. A Survey of Transformers. arXiv 2021, arXiv:2106.04554.

125. Irie, K.; Schlag, I.; Csordás, R.; Schmidhuber, J. Going Beyond Linear Transformers with Recurrent Fast Weight Programmers. arXiv 2021, arXiv:2106.06295.

126. Cahyawijaya, S. Greenformers: Improving Computation and Memory Efficiency in Transformer Models via Low-Rank Approximation. arXiv 2021, arXiv:2108.10808.

127. Katharopoulos, A.; Vyas, A.; Pappas, N.; Fleuret, F. Transformers are RNNs: Fast Autoregressive Transformers with Linear Attention. arXiv 2020, arXiv:2006.16236.

128. Khan, S.; Naseer, M.; Hayat, M.; Zamir, S.W.; Khan, F.S.; Shah, M. Transformers in Vision: A Survey. arXiv 2021, arXiv:2101.01169.

129. Zhu, C.; Ping, W.; Xiao, C.; Shoeybi, M.; Goldstein, T.; Anandkumar, A.; Catanzaro, B. Long-Short Transformer: Efficient Transformers for Language and Vision. arXiv 2021, arXiv:2107.02192.

130. Wang, S.; Li, B.Z.; Khabsa, M.; Fang, H.; Ma, H. Linformer: Self-Attention with Linear Complexity. arXiv 2020, arXiv:2006.04768.

131. Kitaev, N.; Kaiser, Ł.; Levskaya, A. Reformer: The Efficient Transformer. arXiv 2020, arXiv:2001.04451.

132. Shleifer, S.; Weston, J.; Ott, M. NormFormer: Improved Transformer Pretraining with Extra Normalization. arXiv 2021, arXiv:2110.09456.

133. Tlusty, T.; Libchaber, A.; Eckmann, J.-P. Physical model of the sequence-to-function map of proteins. bioRxiv 2016, 069039. [CrossRef] 